\title{
ASYMPTOTIC PROFILES WITH FINITE MASS IN ONE-DIMENSIONAL CONTAMINANT TRANSPORT THROUGH POROUS MEDIA: THE FAST REACTION CASE
}

\author{
By R. E. GRUNDY \\ (Mathematical Institute, University of St Andrews, North Haugh, \\ St Andrews KY16 9SS) \\ C. J. VAN DUIJN
}

(Department of Mathematics and Informatics, Delft University of Technology, PO Box 5031, 2600 GA Delft, The Netherlands)

\author{
and C. N. DAWSON
}

(Department of Mathematical Sciences, Rice University, PO Box 1892, Houston, Texas 77251-1892, USA)

[Received 3 April 1992. Revises 8 October 1992 and 3 February 1993]

\section{SUM MAR Y}

The paper considers the large-time behaviour of positive solutions of the equation

$$
\frac{\partial\left(u+u^{p}\right)}{\partial t}=\frac{\partial^{2} u}{\partial x^{2}}-\frac{\partial u}{\partial x}, \quad p>0
$$

with $-\infty<x<\infty$ and $t \geqslant 0$, for pulse-type initial data. In suitably scaled variables this equation models the one-dimensional flow of a solute through a porous medium with the solute undergoing absorption by the solid matrix of the medium. With the total mass both absorbed and in solution invariant, it is shown that the asymptotic solution depends crucially on the value of $p$. For $p>2$ the solution approaches the symmetric solution of the linear heat equation centred on $x=t$, while for $p=2$ this becomes asymmetric due to the effect of nonlinearity. For $1<p<2$ convection dominates at large time and the solution approaches the form of an asymmetric pulse moving at unit speed along the positive $x$-axis. Diffusion effects are confined to regions near the leading and trailing edges of the pulse. For $0<p<1$ the pulse is still convection-dominated but it no longer moves under a simple translation. Again diffusion only becomes important near the leading and trailing edges. It is shown that for $p \geqslant 2$ the asymptotic solutions are uniformly valid in $x$ but for $0<p<2$ convection-dominated outer solutions have to be supplemented by diffusion boundary layers. In this latter case uniformly-valid composite solutions can be constructed. Finally our asymptotic analyses for the various values of $p$ are compared with numerical solutions of the initial-value problem. 


\section{Introduction}

IN THIS paper we consider the large-time behaviour of solutions of the convection-dispersion equation with nonlinear capacity

$$
\frac{\partial}{\partial t}\left\{u+u^{p}\right\}+\frac{\partial u}{\partial x}-D \frac{\partial^{2} u}{\partial x^{2}}=0, \quad \text { with }(x, t) \in \mathbb{R} \times \mathbb{R}^{+},
$$

subject to the initial condition

$$
u(x, 0)=u_{0}(x) \text { for } x \dot{\in} \mathbb{R} .
$$

Here $p$ and $D$ are positive constants and $u_{0}$ is a non-negative function satisfying the finite-mass property

$$
u_{0}+u_{0}^{p} \in L^{1}(\mathbb{R}) .
$$

The problem (1.1), (1.2) arises as a model for the one-dimensional transport of a solute, with scaled concentration $u \geqslant 0$, through a porous medium. In this model it is assumed that the solute undergoes equilibrium adsorption with the porous matrix. In equation (1.1) the term $u^{p}$ denotes the scaled adsorbed concentration. The integrability condition (1.3) implies that initially the total mass, both in solution and adsorbed, is finite.

For the purposes of this paper the work is organized as follows.

1. Introduction

2. The model

3. Some analytical remarks

4. The asymptotic solution

4.1 The outer solutions

4.1.1 The case $p>2$

4.1.2 The case $1<p \leqslant 2$

4.1.3 The case $p<1$

4.2 The uniformity of the outer solutions: the translation-layer solutions

4.2.1 The case $1<p<2$

4.2.2 The case $0<p<1$

4.3 Construction of uniformly-valid solutions for $0<p<2$

5. The numerical method

6. Conclusions

References

Appendix A Error analysis for $p=3$ and $p=2$

Appendix B Solutions to a boundary value problem

Appendix $C$ The solution for $D=0$ when $p=\frac{1}{2}$ and $p=\frac{3}{2}$

In section 2 we discuss the physical background of the problem and derive equation (1.1). In section 3 some analytical properties of solutions are given, such as the occurrence of free boundaries when $p \in(0,1)$ and the large-time behaviour when the initial distribution satisfies either $u_{0}(-\infty)=1, u_{0}(+\infty)=0$ 
(convergence towards a travelling wave) or $u_{0}(-\infty)=0, u_{0}(+\infty)=1$ (convergence towards a rarefaction wave). We also compare our findings with the analytical results of Escabedo, Vazquez and Zuazua (1). Next, in section 4, the asymptotic form as $t \rightarrow \infty$ of pulse-type solutions satisfying

$$
\left(u+u^{p}\right)(\cdot, t) \in L^{1}(\mathbb{R}) \text { for all } t>0
$$

is derived. We discuss first the outer solutions in section 4.1 and thereafter, in section 4.2, the boundary-layer solutions which occur for $0<p<1$ and $1<p<2$. We also compare the asymptotic profiles with the numerical solution of problem (1.1), (1.2). The algorithm, which is discussed in section 5, is based on a higher-order Godunov approach, which makes it possible to compute solutions of (1.1) with $D$ small, or even with $D=0$. In section 6 the concluding remarks are given as well as an interpretation of the results in terms of the physical model.

\section{The model}

In this section we formulate a model for the one-dimensional transport of a one-species contaminant through a porous medium. To begin with, we consider the flow of an incompressible fluid through a homogeneous and saturated porous medium. We shall assume that the flow is steady, macroscopically one-dimensional and directed along what is chosen to be the positive $x$-axis. It is characterized by the volumetric flux, also known as specific discharge, which will be denoted by $q(\mathrm{~m} / \mathrm{s})$.

In the fluid a one-species solute is present at tracer-level concentration $C$ $\left(\mathrm{mol} / \mathrm{ml}^{3}\right)$. This means that the flow is independent of the solute distribution. We shall therefore take $q$ to be a known positive constant.

If no adsorption reactions occur between the solute and the surrounding solid part of the porous medium, then the transport is determined by convection, molecular diffusion and mechanical dispersion; see for instance Bear (2) or Freeze and Cherry (3). However, if adsorption reactions do take place, this has to be taken into account when describing the transport process. In this reactive case we denote by $S$ (mol/ $/ \mathrm{kg}$ porous material) the adsorbed concentrations. If the boundary and flow conditions are such that both $C$ and $S$ can be assumed to be constant in planes perpendicular to the $x$-axis, implying that $C=C(x, t)$ and $S=S(x, t)$, then mass conservation yields the expression (see, for example, Bolt (4)).

$$
\frac{\partial}{\partial t}\{\theta C+\rho S\}+\frac{\partial}{\partial x}\left\{q C-D \frac{\partial C}{\partial x}\right\}=0
$$

where $t$ and $x$ denote, respectively, time and space coordinates. Here $\theta(-)$ is the porosity of the porous material, $\rho\left(\mathrm{kg} / \mathrm{m}^{3}\right)$ its bulk mass density and $D$ $\left(\mathrm{m}^{2} / \mathrm{s}\right)$ is the coefficient of hydrodynamic dispersion, which is the sum of molecular diffusion and mechanical dispersion. 
All coefficients in (2.1) can be considered as being constant and positive. The term $\rho(\partial S / \partial t)$ in (2.1) represents the rate of change of concentration on the porous matrix due to adsorption or desorption.

Now we consider the adsorption process. In general, the relation between the concentration in the fluid and the adsorbed concentration is described by a first-order ordinary differential equation of the form

$$
\frac{\partial S}{\partial t}=k f(C, S),
$$

where $k>0\left(\mathrm{~s}^{-1}\right)$ is the rate parameter and $f(\mathrm{~mol} / \mathrm{kg}$ porous material $)$ is the reaction rate function. In (5) general rate functions are considered, some of which are discussed below together with some of their properties.

If we can solve the equation

in the form

$$
f(C, S)=0
$$

$$
S=\psi(C)
$$

then we call $\psi$ the adsorption isotherm. In many cases, rate functions and isotherms satisfy the following monotonicity properties

$$
\begin{gathered}
f(C, S)>(<) 0 \text { if and only if } S<(>) \psi(C), \\
\psi(0)=0 \text { and } \psi \text { strictly increasing and smooth for } C>0
\end{gathered}
$$

The isotherms $\psi$ are sometimes classified according to their behaviour near $C=0$. We say that

1. $\psi$ is of Langmuir ( $L$-type) if $\psi$ is strictly concave near $C=0$ and $\psi^{\prime}(0+)<\infty$;

2. $\psi$ is of Freundlich ( $F$-type) if $\psi$ is strictly concave near $C=0$ and $\psi^{\prime}(0+)=\infty$;

3. $\psi$ is of convex ( $S$-type) if $\psi$ is strictly convex near $\mathrm{C}=0$.

The distinction between these classes is of importance, because different isotherms may give different transport behaviours for the solutes. In mathematical terms, the regularity and global behaviour of the solutions may be different if $\psi$ is taken from these different classes. This is clearly the case when considering the asymptotic profiles in sections 4 and 5 .

Well-known examples of isotherms are

1. the Langmuir isotherm, where

and

$$
\psi(C)=\frac{K_{1} C}{1+K_{2} C} \quad \text { with } K_{1}, K_{2}>0
$$

2. the Freundlich isotherm, where

$$
\psi(C)=K_{3} C^{p} \quad \text { with } K_{3}, p>0 .
$$

In (2.6) the isotherm is of $F$-type if $0<p<1$ and of $S$-type if $p>1$. The case 
when $0<p<1$ occurs in many practical situations, although values for which $p>1$ have been used (see, for example, van Genuchten and Cleary (6)).

In this paper we restrict ourselves to the case of fast reactions or equivalently equilibrium adsorption. Mathematically this is achieved by letting $k \rightarrow \infty$ in (2.2). As a result we have

$$
S=\psi(C)
$$

see also (2.3). The convergence process in which $k \rightarrow \infty$ is discussed in detail by Knabner (7) and van Duijn and Knabner (8, 9). From a physical viewpoint this limit implies that the adsorption reactions are very rapid in comparison with the flow velocity, so that the adsorbed concentration instantaneously follows the variations of the solute concentration.

We also restrict ourselves with respect to the choice of the isotherm. To be specific we shall consider the case of Freundlich isotherms only, whence the transport equation (2.1) becomes

$$
\frac{\partial}{\partial t}\left\{\theta C+\rho K_{3} C^{p}\right\}+\frac{\partial}{\partial x}\left\{q C-D \frac{\partial C}{\partial x}\right\}=0 .
$$

We shall consider solutions of this equation in the half-space

$$
Q=\{(x, t):-\infty<x<\infty, t>0\},
$$

and impose the initial condition

$$
C(x, 0)=C_{0}(x)
$$

at $t=0$. To eliminate the constants from (2.8) we apply the following scaling and redefinition: for $p=1$,

$$
u=C, \quad t=2 \frac{q}{\theta}\left(1+\frac{\rho K_{3}}{\theta}\right)^{-1} t, \quad x=x, \quad D=\frac{D}{q} ;
$$

for $p \neq 1$,

$$
u=\left(\frac{\rho K}{\theta}\right)^{1 /(p-1)} C, \quad t=\frac{q}{\theta} t, \quad x=x, \quad D=\frac{D}{q} .
$$

This leads to the initial-value problem (for all $p>0$ )

where

$$
\begin{gathered}
\frac{\partial}{\partial t}\left\{u+u^{p}\right\}+\frac{\partial u}{\partial x}-D \frac{\partial^{2} u}{\partial x^{2}}=0 \quad \text { for }(x, t) \in Q, \\
u(x, 0)=u_{0}(x) \text { for } x \in \mathbb{R},
\end{gathered}
$$

$$
u_{0}(x)= \begin{cases}C_{0}(x) & \text { for } p=1 \\ \left(\frac{\rho K_{3}}{\theta}\right)^{1 /(p-1)} C_{0}(x) & \text { for } p \neq 1\end{cases}
$$


We shall consider the large time-behaviour of solutions of the initial-value problem (2.11), (2.12) for the case where $u_{0}$ is a pulse satisfying

$$
u_{0}( \pm \infty)=0, \quad u_{0} \geqslant O(\not \equiv 0) \text { on } R \text {. }
$$

In particular we shall require that

$$
u_{0}+u_{0}^{p} \in L^{1}(\mathbb{R}),
$$

so that the total mass of adsorbed and dissolved concentration is finite.

We note that we could have chosen a scaling which also eliminates the constant $D$ from equation (2.11). To be specific define

$$
x=\frac{x}{D}, \quad t=\frac{t}{D}
$$

to give

$$
\frac{\partial\left(u+u^{p}\right)}{\partial t}=\frac{\partial^{2} u}{\partial x^{2}}-\frac{\partial u}{\partial x},
$$

which is the equation we actually consider in section 4 .

However, keeping $D$ in front of the second derivative allows us to consider the hyperbolic limit $D \downarrow 0$. We come back to this point in the concluding remarks of section 6 .

We finally observe that in many cases of practical interest $\rho K_{3} / \theta \gg 1$. This means that the implication of the scaling is quite different for $p<1, p=1$ and $p>1$. One has to bear this in mind when comparing solutions of $(2.11),(2.12)$ for different values of $p$.

\section{Some analytical remarks}

We first set

$$
\beta(u)=u+u^{p} \quad \text { for } u \geqslant 0,
$$

and write equation (2.11) as

$$
\frac{\partial}{\partial t} \beta(u)+\frac{\partial u}{\partial x}-\frac{\partial^{2} u}{\partial x}=0 .
$$

We have put $D=1$ in (3.2) which, as we note from (2.19), can be done without loss of generality. Equation (3.2) is a nonlinear second-order equation of parabolic type. Since $\beta^{\prime}(u)$ may tend to infinity when $u$ tends to zero $(p<1)$, equation (3.2) can degenerate at points where its solution vanishes. Therefore we cannot expect the initial-value problem (2.11), (2.12) to have classical solutions for values of $p$ belonging to the interval $(0,1)$.

Writing

$$
w=\beta(u) \text { and } u=\varphi(w),
$$

where $\varphi=\beta^{-1}$ denotes the inverse of the function $\beta$, we obtain for $w$ the trans- 
formed initial-value problem

$$
\begin{gathered}
\frac{\partial w}{\partial t}+\frac{\partial \varphi(w)}{\partial x}-\frac{\partial^{2} \varphi(w)}{\partial x^{2}}=0 \quad \text { for }(x, t) \in Q, \\
w(x, 0)=w_{0}(x)=u_{0}(x)+u_{0}^{p}(x) \quad \text { for } x \in \mathbb{R} .
\end{gathered}
$$

The existence and uniqueness theory for the problem (3.4), (3.5) is well known and can be found in Gilding (10). We can therefore use these results to make some statements about the solvability of $(2.11),(2.12)$ for $u$. If we assume that $u_{0}$, and hence $w_{0}$, is such that

$$
u_{0} \in C(\mathbb{R}),
$$

then there exist numbers $-\infty<a_{1}<a_{2}<\infty$ such that

$$
u_{0}(x) \begin{cases}=0, & -\infty<x \leqslant a_{1}, \\ >0, & a_{1}<x<a_{2}, \\ =0, & a_{2} \leqslant x<\infty .\end{cases}
$$

Then we can make the following statements.

(i) If $p \geqslant 1$ then the problem (2.11), (2.12) has a unique classical solution $u \in C^{\infty}(Q) \cap C(\bar{Q})$ which satisfies $u>0$ in $Q$;

(ii) If $p \in(0,1)$ then $(2.11),(2.12)$ has a unique weak (distributional) solution $u \in C(\bar{Q})$. At points where $u>0$, the solution is smooth (that is, $\left.u \in C^{\infty}(\{u>0\})\right)$ and satisfies the equation classically. Moreover, there exist functions (for $i=1,2) s_{t} \in C\left([0, \infty)\right.$ ), satisfying $s_{i}(0)=a_{i}$ and $-\infty<s_{1}(t)<\infty$ for all $t>0$, which form the support of $u$ in the $x, t$ plane; that is, $u(x, t)>0$ if and only if $x \in\left(s_{1}(t), s_{2}(t)\right)$ for every $t \geqslant 0$.

The functions $s_{i}$ are called interfaces or free boundaries and they occur only in the degenerate case $p \in(0,1)$.

In this paper we are interested in the large-time behaviour of solutions of the problem (2.11), (2.12). First we make some statements about previous work on equation (3.2) which, to the authors' knowledge, has dealt exclusively with initial data satisfying

where either

$$
u_{0}( \pm \infty)=u_{ \pm},
$$

$$
0 \leqslant u_{-}<u_{+}<\infty
$$

or

$$
\infty>u_{-}>u_{+} \geqslant 0 .
$$

We note that (3.2) has travelling-waves solutions $u(x, t)=f(x-c t)$, which satisfy $f( \pm \infty)=u_{ \pm}$, where $u_{+}$and $u_{-}$satisfy (3.8a) if $p>1$ and (3.8b) if 
$p \in(0,1)$ and where the wave speed $c$ is given by

$$
c=\frac{u_{+}-u_{-}}{\beta\left(u_{+}\right)-\beta\left(u_{-}\right)} .
$$

The stability of these travelling waves follows from a result of Osher and Ralston (11). They employ a contraction property of the semigroup associated with the transformed problem (3.4), (3.5) to prove convergence, as $t \rightarrow \infty$ in $L^{1}(\mathbb{R})$, towards a suitably shifted travelling wave.

The large-time behaviour for the cases where no travelling waves exist, that is,

$$
p>1 \text { and (3.8b) }
$$

or

$$
p \in(0,1) \text { and (3.8a) }
$$

was considered by van Duijn and De Graaf (12). For this parameter choice, the contaminant profiles become flatter as time increases. In fact it was shown that $\beta(u(\cdot, t))$ converges to the transformed solution $u^{*}$ of the reduced hyperbolic problem

$$
\begin{aligned}
\frac{\partial}{\partial t} \beta(u)+\frac{\partial u}{\partial x} & =0 \quad \text { for }(x, t) \in Q \\
u(x, 0) & = \begin{cases}u_{-}, & x<0, \\
u_{+}, & x>0 .\end{cases}
\end{aligned}
$$

The function $u^{*}$ is a rarefaction wave with $u^{*}=u^{*}(x / t)$. Since $\beta$ is given by (3.1), it is easy to obtain explicit expressions for $u^{*}$. The convergence analysis gives an estimate for decay rate of $\left\|\beta(u(\cdot, t))-\beta\left(u^{*}(\cdot, t)\right)\right\|_{L \infty(\mathbf{R})}$ as $t \rightarrow \infty$.

We now make some remarks about the solution to (2.11), (2.12) and the procedures used to find the asymptotic solutions. We first note that the solutions have mass conservation, that is, for all $t>0$

$$
\begin{aligned}
\int_{\mathbf{R}}\left\{u+u^{p}\right\}(x, t) d x & =\int_{\mathbf{R}}\left(u_{0}+u_{0}^{p}\right)(x) d x \\
& =M, \text { say }
\end{aligned}
$$

This property, together with scaling arguments, plays a crucial role in establishing the asymptotic solutions.

In constructing the asymptotic solutions of section 4 we use the following intuitive ideas. In the degenerate case, $p \in(0,1)$, for $t \rightarrow \infty$ we may write

$$
u+u^{p} \sim u^{p}
$$

and consider the simplified equation

$$
\frac{\partial\left(u^{p}\right)}{\partial t}+\frac{\partial u}{\partial x}-\frac{\partial^{2} u}{\partial x}=0
$$


to obtain the asymptotic limit. This procedure is formalized in detail in section 4.1.3.

When $p=1$, we have the linear equation

$$
2 \frac{\partial u}{\partial t}+\frac{\partial u}{\partial x}-\frac{\partial^{2} u}{\partial x^{2}}=0
$$

which has the asymptotic profile

$$
u(x, t) \rightarrow \frac{M}{(2 \pi t)^{\frac{t}{t}}} \exp \left\{-\frac{1}{2 t}\left(x-\frac{t}{2}\right)^{2}\right\} \quad \text { as } t \rightarrow \infty .
$$

When $p>1$ the situation becomes more subtle. Both the numerical evidence and the solution in the hyperbolic limit $(D \downarrow 0)$ suggest that we first transform to the moving coordinates

$$
t=t \quad \text { and } \quad \xi=x-t
$$

which gives the equation, now with nonlinear convection,

Next we write

$$
\frac{\partial}{\partial t}\left(u+u^{p}\right)-\frac{\partial u^{p}}{\partial \xi}-\frac{\partial^{2} u}{\partial \xi^{2}}=0 .
$$

$$
u+u^{p} \sim u
$$

as $t \rightarrow \infty$ and obtain the equation

$$
\frac{\partial u}{\partial t}-\frac{\partial u^{p}}{\partial \xi}-\frac{\partial^{2} u}{\partial \xi^{2}}=0
$$

This procedure is also formalised in section 4 , where we distinguish the following cases. For $1<p<2$, we show in section 4.1.2 that convection terms in (3.18) dominate diffusion as $t \rightarrow \infty$ and the asymptotic profile becomes, in the appropriate variables, a semi- $N$-wave.

For $p=2$ the convection and diffusion terms in (3.18) balance in the large-time limit, resulting in a Burgers equation for which a self-similar limit profile exists which the solution approaches. This is also given in section 4.1.2.

For $p>2$ we show that the diffusion term in (3.18) dominates convection and that the asymptotic profiles are now symmetric self-similar solutions of the heat equation; see section 4.1.1.

The asymptotic forms discussed above are called outer solutions. We note here that convergence (in the $L^{1}$ sense) of solutions of (3.18) towards these outer solutions for $p>1$ was proved by Escabedo, Vasquez and Zuazua (1) and Escabedo and Zuazua (13). However for $1<p<2$ there are two defects associated with the outer solutions. First they are not continuous and secondly they do not have unbounded support. It is necessary therefore to supplement the outer solutions by boundary-layer solutions which are valid in thin regions 
near points of discontinuous behaviour of the outer solution. Except for one case, we can solve the boundary-layer equations explicitly. These solutions can be used to do two things: first to render the outer solution continuous and secondly to give the solution unbounded support. Boundary layers have also to be inserted when $0<p<1$ to smooth out the outer solution and locate the position of the free boundaries which occur since the outer solution predicts, erroneously, a stationary interface at one end of its support.

\section{The asymptotic solution}

\subsection{The outer solutions}

In this section we construct the large-time solution of the scaled equation

$$
\frac{\partial\left(u+u^{p}\right)}{\partial t}=\frac{\partial^{2} u}{\partial x^{2}}-\frac{\partial u}{\partial x}, \quad p>0,(x, t) \in Q
$$

with pulse-type initial conditions satisfying (2.13). Our approach is to a large extent intuitive in the sense that we postulate an analytic form for the solution and then deduce for what range of values of $p$ this type of solution is expected to occur. This idea has been successfully used in a number of papers devoted to large-time asymptotics for nonlinear diffusion and related equations, particularly for pulse-type initial data with either bounded or unbounded support; see for example Grundy $(14,15,16)$.

The nature of the limiting solution depends on the value of the parameter $p$ and reflects the relative importance of the various terms in (4.1) as $t$ becomes large. As will become apparent later on, it is natural to take the cases $0<p<1$, $1<p<2$ and $p>2$ separately. Let us take $p>2$ first.

4.1.1 The case $p>2$. We start off by transforming equation (4.1), using the moving coordinate system

$$
(t, \xi=x-t)
$$

with $u=u(\xi, t)$, giving

$$
\frac{\partial}{\partial t}\left(u+u^{p}\right)=\frac{\partial^{2} u}{\partial \xi^{2}}+\frac{\partial\left(u^{p}\right)}{\partial \xi} .
$$

We would expect the spread of the solution to be incorporated by using the similarity variable

$$
\eta=\frac{\xi}{t^{\delta}}=\frac{x-t}{t^{\delta}},
$$

where $\delta>0$, together with the change of dependent variable

$$
u(\xi, t)=t^{a} v(\eta, t)
$$


with $\alpha<0$ to simulate temporal decay. In the new variables (4.3) now becomes

$$
\begin{aligned}
&\left\{t \frac{\partial v}{\partial t}+\alpha v-\delta \eta \frac{\partial v}{\partial \eta}\right\}+t^{\alpha(p-1)}\left\{t \frac{\partial\left(v^{p}\right)}{\partial t}+\alpha p v^{p}-\delta \eta \frac{\partial\left(v^{p}\right)}{\partial \eta}\right\} \\
&=t^{1-2 \delta} \frac{\partial^{2} v}{\partial \eta^{2}}+t^{\alpha(p-1)+(1-\delta)} \frac{\partial\left(v^{p}\right)}{\partial \eta},
\end{aligned}
$$

which we write as $A+B=C+D$.

The immediate aim of the analysis is to determine the unknown indices $\alpha$ and $\delta$. To assist us in this task we turn to the time invariant (3.13), namely

$$
M=\int_{-\infty}^{\infty}\left\{u+u^{p}\right\} d x
$$

which, in terms of $\eta$ and $v$, can be written as

Thus, for $p>1$

$$
M=\int_{-\infty}^{\infty}\left\{t^{\alpha+\delta} v+t^{\alpha p+d} v^{p}\right\} d \eta
$$

$$
M \sim t^{a+\delta} \text { as } t \rightarrow \infty
$$

and hence for $M$ to be invariant in time,

$$
\delta=-\alpha .
$$

Remembering that we are seeking solutions in the limit $t \rightarrow \infty, \eta=O(1)$, we now assume that $v$ and its derivatives with respect to $\eta$ together with $t \partial v / \partial t$ are bounded in this limit. With this assumption it is clear that for $p>1$ and $\alpha<0$ the term $A$ dominates the left-hand side of (4.6) as $t \rightarrow \infty$. Two possibilities now emerge; either I: $C$ dominates $D$ on the right-hand side of (4.6) and balances asymptotically with $A$ or II: $D$ dominates $C$ on the right-hand side and balances asymptotically with $A$. By the term asymptotic balancing we mean asymptotically equivalent as $t \rightarrow \infty$. The first possibility (I) requires that

and

$$
\delta=\frac{1}{2}
$$

$$
\alpha(p-1)+1-\delta<0 .
$$

With $\alpha=-\frac{1}{2}$ from (4.9), condition (4.11) is simply

whence (4.6) becomes

$$
p>2 \text {, }
$$

$$
\begin{aligned}
t \frac{\partial v}{\partial t}-\frac{1}{2}\left(v+\eta \frac{\partial v}{\partial \eta}\right)+t^{-(p-1) / 2}\left\{t \frac{\partial\left(v^{p}\right)}{\partial t}-\right. & \left.\frac{p}{2} v^{p}-\frac{\eta}{2} \frac{\partial\left(v^{p}\right)}{\partial \eta}\right\} \\
& =\frac{\partial^{2} v}{\partial \eta^{2}}+t^{-(p-2) / 2} \frac{\partial\left(v^{p}\right)}{\partial \eta}
\end{aligned}
$$


We now expand

$$
v(\eta, t)=v_{0}(\eta)+o(1)
$$

such that $t \partial v / \partial t=o(1)$, in the limit $t \rightarrow \infty, \eta=O(1)$ and substitute into (4.13). Collecting leading-order terms gives

$$
v_{0}^{\prime \prime}+\frac{1}{2}\left(\eta v_{0}^{\prime}+v_{0}\right)=0,
$$

where primes denote differentiation with respect to $\eta$. The general solution of this equation is

$$
v_{0}=K_{1} e^{-\eta^{2} / 4}-K_{2} e^{-\eta^{2} / 4} \int_{\eta}^{\infty} e^{s^{2} / 4} d s=K_{1} e^{-\eta^{2} / 4}+O\left(\eta^{-1}\right) \text { as } \eta \rightarrow \infty
$$

where $K_{1}$ and $K_{2}$ are arbitrary constants. Bearing in mind that mass invariance, for $p>2$, in the form $(4.8)$ requires $v_{0}(\eta)$ be integrable on $(-\infty, \infty)$, we must put $K_{2}=0$ to give

$$
v_{0}=K_{1} e^{-\eta^{2} / 4}
$$

Substituting (4.14) into (4.8), with (4.15), the leading-order result gives

$$
M=K_{1} \int_{-\infty}^{\infty} e^{-\eta^{2} / 4} d \eta \text { or } K_{1}=\frac{M}{2 \sqrt{\pi}} .
$$

Thus we have shown that for $p>2$,

$$
u(x, t)=\frac{M}{2 \sqrt{ } \pi} t^{-\frac{1}{2}} e^{-(x-t)^{2} / 4 t}\{1+o(1)\}
$$

as $t \rightarrow \infty,(x-t) / 2 \sqrt{ } t=O(1)$. This result is uniform in $x$.

It is instructive at this stage to compare the leading-order behaviour of (4.16) with the numerical computations. In Fig. 1 we plot $v(\eta, t) \equiv t^{1 / 2} u(x, t)$ as a function of $\eta$ for $p=3$ and the initial data

$$
u_{0}(x)=H(x+1)-H(x-1)
$$

for various values of $t$. Here $H(x)$ is the Heaviside function. So with $M=4$ from (4.7) the results show a slow but evident convergence to the asymptotic profile

$$
\frac{2}{\sqrt{\pi}} e^{-\pi^{2} / 4}
$$

the slowness due presumably to the neglect of the dominant convective error term $t^{-(p-2) / 2} \partial\left(v^{p}\right) / \partial \eta$ in (4.13). If we include this term in a first-order error analysis then we show in Appendix $A$ that for $p=3$ and $t \rightarrow \infty$

$$
v(\eta, t)=v_{0}(\eta)+\frac{4 t^{-1 / 2} \log t}{\pi \sqrt{ } 3} v_{0}^{\prime}(\eta)+O\left(t^{-1 / 2}\right)
$$




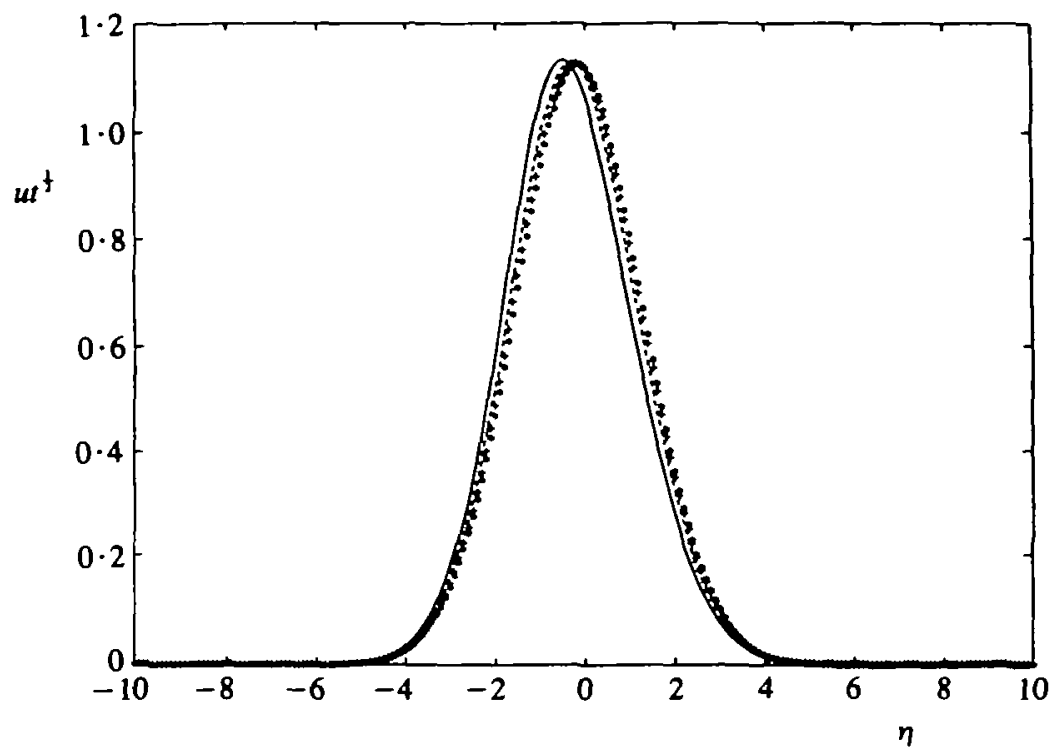

FIG. 1. Convergence of the numerical solution to the limiting profile for the case $p=3$. $u t^{1 / 2}$ plotted as a function for $\eta$ for $-t=80, \cdots t=320$, $++t=1000, * * * t=2000$

or, equivalently, that

where

$$
v(\eta, t)=v_{0}\left(\eta_{1}\right)+O\left(t^{-1 / 2}\right)
$$

$$
\eta_{1}=\eta+\frac{4 t^{-1 / 2} \log t}{\pi \sqrt{3}} .
$$

The utility of this device is shown in Fig. 2, where the numerical solution is represented as a function of $\eta_{1}$. The accelerated convergence to

is clearly evident.

$$
\frac{2}{\sqrt{\pi}} \exp \left(-\eta_{1}^{2} / 4\right)
$$

We recall here the results of Escabedo et al. (1) and Escabedo and Zuazua (13), who proved that for $p>2$ the finite-mass solutions of (4.3), after neglecting the time derivative of $u^{p}$, converge in the $L^{1}$ sense to the solution of the heat equation, obtained by disregarding the derivative $\partial\left(u^{p}\right) / \partial \xi$ in (3.17). Our analysis includes this term as $t \rightarrow \infty$ and enables us to improve the convergence to the asymptotic profile.

4.1.2 The case $1<p \leqslant 2$. We now turn to the second (II) possibility in (4.6). By equating powers of $t$ as before we find that

$$
\alpha(p-1)+1-\delta=0
$$




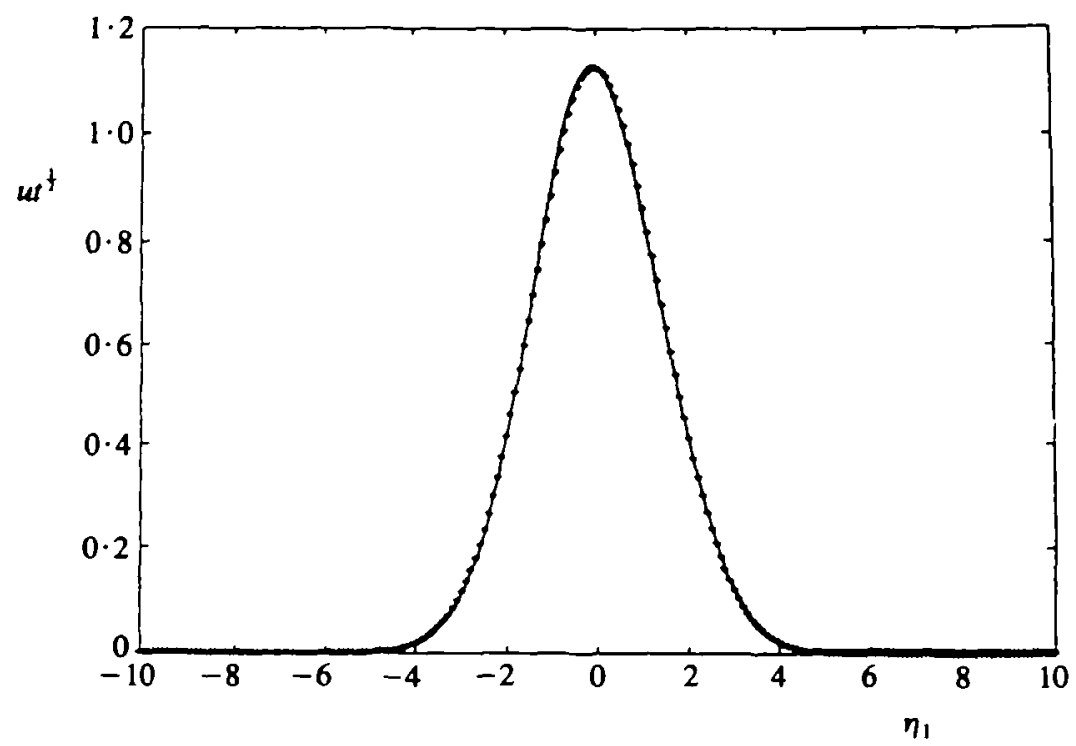

FIG. 2. Convergence of the numerical solution to the limiting profile for the case $p=3$. $u t^{1 / 2}$ plotted as a function of the modified variable $\eta_{1}$ for $-t=200, \cdots t=500,+++t=1000, * * * t=1500$

and

$$
1-2 \delta<0
$$

Hence, using (4.9), (4.21) yields

$$
\alpha=-\frac{1}{p}, \quad \delta=\frac{1}{p}
$$

and the inequality (4.22) becomes

$$
p<2 \text {. }
$$

With these values of $\alpha$ and $\delta(4.6)$ can be written as

$$
\begin{aligned}
t \frac{\partial v}{\partial t}-\frac{1}{p}\left(v+\eta \frac{\partial v}{\partial \eta}\right)+t^{-(p-1) / p}\left\{t \frac{\partial\left(v^{p}\right)}{\partial t}-\right. & \left.v^{p}-\frac{\eta}{p} \frac{\partial\left(v^{p}\right)}{\partial \eta}\right\} \\
& =t^{-(2-p) / p} \frac{\partial^{2} v}{\partial \eta^{2}}+\frac{\partial\left(v^{p}\right)}{\partial \eta} .
\end{aligned}
$$

We now expand

$$
v=v_{0}(\eta)+o(1)
$$

as $t \rightarrow \infty, \eta=O(1)$, with $t(\partial v / \partial t)=o(1)$, which we call the outer expansion. Leading-order terms in (4.25) now give

$$
\left(v_{0}+\eta v_{0}^{\prime}\right)+p\left(v_{0}^{p}\right)^{\prime}=0,
$$


where again primes denote differentiation with respect to $\eta$. The general solution of this equation is

$$
v_{0}^{p}+\frac{\eta}{p} v_{0}=C,
$$

where $C$ is an arbitrary constant. In order to fix the value of $C$ in this solution we use the following argument. For $C<0 v_{0}(\eta)$ is double-valued with

$$
\eta \leqslant-p^{2}(-C)^{1 / p} /(p-1)^{(p-1) / p}
$$

a solution which we reject. On the other hand for $C>0 v_{0}(\eta)$ is single-valued on $-\infty<\eta<\infty$ but as $\eta \rightarrow+\infty$

$$
v_{0} \sim p C / \eta \text {. }
$$

Since mass invariance requires $v_{0}(\eta)$ be integrable on $(-\infty, \infty)$, it follows that $C$ cannot be positive. Thus we must take $C=0$ and the solution for $v_{0}$ is simply

$$
v_{0}=\left(-\frac{\eta}{p}\right)_{+}^{1 /(p-1)} .
$$

The solution (4.27) is defined for all $\eta \in \mathbb{R}$ but gives a solution with infinite mass. To ensure a finite mass $M$ as given by (4.8) we must restrict the range of validity of (4.27) to the finite interval $\eta_{1} \leqslant \eta \leqslant 0$, setting $v_{0}$ identically zero outside this interval. This leads to an outer solution with a discontinuity at $\eta_{1}$. Substituting (4.26) into (4.8), with (4.27), gives to leading order

and hence

$$
M=\int_{\eta 1}^{0}\left(\frac{-\eta}{p}\right)^{1 /(p-1)} d \eta
$$

$$
\eta_{1}=-p\left(\frac{M}{p-1}\right)^{(p-1) / p}<0 .
$$

In terms of $x$ and $t$ therefore we have shown that for $1<p<2$

$$
u(x, t)=t^{-1 / p}\left(\frac{t-x}{p t^{1 / p}}\right)^{1 /(p-1)}\{1+o(1)\}
$$

as $t \rightarrow \infty,(x-t) / t^{1 / p}=O(1)$. The condition $\eta_{1} \leqslant \eta \leqslant 0$ requiring in (4.29) that

$$
\eta_{1} t^{1 / p} \leqslant x-t<0 .
$$

It is convenient at this stage to consider the borderline case $p=2$. In this event the terms $A, C$ and $D$ are asymptotically equivalent at $t \rightarrow \infty, \eta=O(1)$, whence

Expanding

$$
\alpha=-\delta=-\frac{1}{2} \text {. }
$$

$$
v=v_{0}(\eta)+o(1)
$$

with $t \partial v / \partial t=o(1)$ as before, yields a second-order differential equation for $v_{0}$ 
which has the solution

$$
v_{0}(\eta)=\frac{e^{-\eta^{2} / 4}}{K+\sqrt{ } \pi \operatorname{erf}(\eta / 2)},
$$

where $K$ is an arbitrary constant given by the asymptotic mass-invariance condition

$$
M=\int_{-\infty}^{\infty} v_{0}(\eta) d \eta
$$

This condition gives

$$
K=\sqrt{ } \pi\left(e^{M}+1\right) /\left(e^{M}-1\right) .
$$

Again we can compare this asymptotic result with the full numerical solution. With $u_{0}$ given by (4.17), $v(\eta, t)=t^{1 / p} u(x, t)$ converges rather slowly to (4.26) due to the neglected term of $O\left(t^{-1 / 2}\right)$ in (4.13). To include this term we can go through a procedure similar to the one for $p>2$. In Appendix $A$ we expand

$$
v(\eta, t)=v_{0}(\eta)-0.0639 t^{-1 / 2}(\log t) v_{0}^{\prime}(\eta)+O\left(t^{1 / 2}\right),
$$

suggesting that we may write

$$
v(\eta, t)=v_{0}\left(\eta_{1}\right)+O\left(t^{1 / 2}\right),
$$

where $\eta_{1}=\eta-0.0639 t^{-1 / 2} \log t$. The numerical solution is represented as a function of $\eta_{1}$ in Fig. 3, where a somewhat faster convergence to the asymptotic profile is evident.

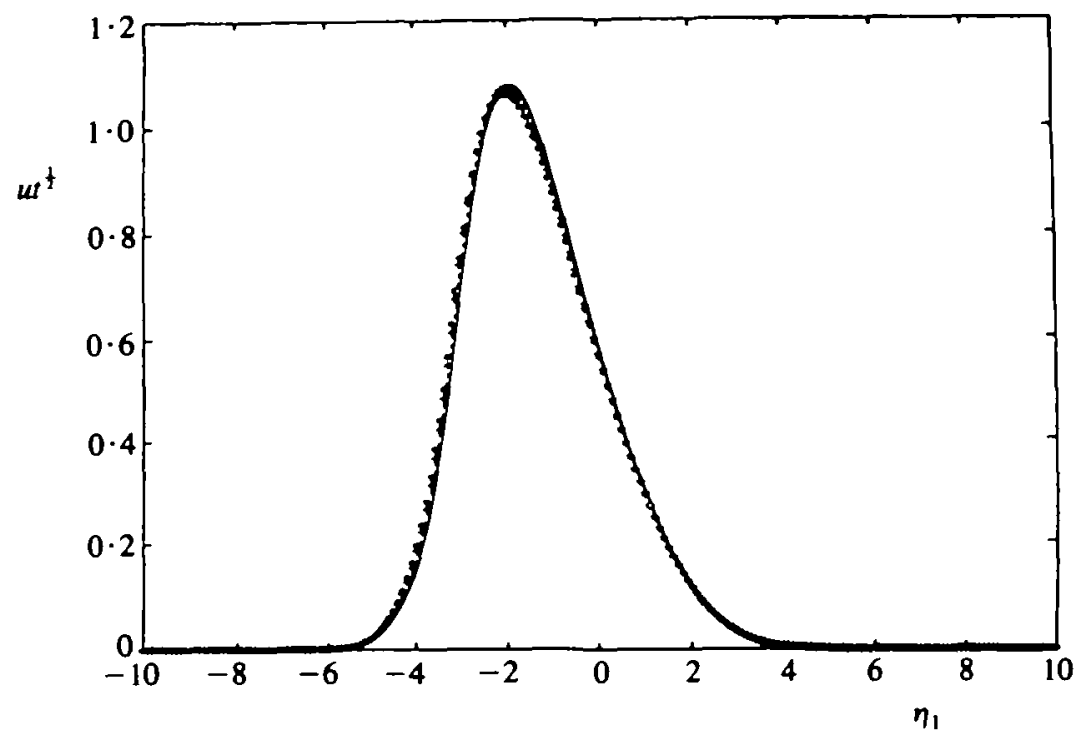

FiG. 3. Convergence of the numerical solution to the limiting profile for the case $p=2$. $u t^{1 / 2}$ plotted as a function of the modified variable $\eta_{1}$ for $-t=500, \cdots t=1000,+++t=1500, * * * t \sim 3000$ 
Finally we note that (4.26) agrees with the convergence result of Escabedo et al. (1) for these cases. This can be understood if one disregards the time derivative of $u^{p}$ in equation (4.3). Also note that the function $v_{0}$ in (4.26) is a self-similar solution of Burgers's equation ((3.19) with $p=2)$.

4.1.3 The case $p<1$. Our intuition suggest that we adopt a different strategy by considering a similarity variable with no translation. This is confirmed by the computations and the propagation properties of the profile in the hyperbolic limit, see Appendix C. So now we put

$$
\eta=\frac{x}{t^{v}}, \quad v>0
$$

with

$$
u(x, t)=t^{\beta} v(\eta, t), \quad \beta<0 .
$$

Making the change of variable in (1.1) gives

$$
\begin{aligned}
t^{\beta(1-p)}\left\{t \frac{\partial v}{\partial t}+\beta v-v \eta \frac{\partial v}{\partial \eta}\right\} & +\left\{t \frac{\partial\left(v^{p}\right)}{\partial t}+\beta p v^{p}-v \eta \frac{\partial\left(v^{p}\right)}{\partial \eta}\right\} \\
& =t^{\beta(1-p)+1-2 v} \frac{\partial^{2} v}{\partial \eta^{2}}-t^{\beta(1-p)+1-v} \frac{\partial v}{\partial \eta} .
\end{aligned}
$$

Clearly since $\beta<0$ and $p<1, B$ dominates the left-hand side of (4.35) as $t \rightarrow \infty$. The question is with which term on the right-hand side does $B$ balance. It turns out that the only consistent possibility is that $D$ dominates $C$ and takes up the asymptotic balance with $B$. This requires that

$$
\beta(1-p)+1-v=0
$$

and

$$
\beta(1-p)+1-2 v<0 .
$$

The second equation relating $\beta$ and $v$ in addition to (4.36) is obtained from the mass-invariance result (4.7). Writing this in terms of $\eta$ and $v$ from (4.33) and (4.34) we have

$$
M=\int_{-\infty}^{\infty}\left\{t^{\beta+v} v+t^{\beta p+v} v^{p}\right\} d \eta
$$

and, for $p<1$ with $\beta<0$,

$$
M \sim t^{\beta p+\nu} \text { as } t \rightarrow \infty .
$$

Hence the invariance of $M$ requires that

which, along with (4.36), gives

$$
v=-\beta p,
$$

$$
\beta=-1 \text { and } v=p .
$$


With these values of $\beta$ and $v,(4.35)$ becomes

$$
t \frac{\left(\partial v^{p}\right)}{\partial t}-p\left\{v^{p}+\eta \frac{\partial\left(v^{p}\right)}{\partial \eta}\right\}+t^{-(1-p)}\left\{t \frac{\partial v}{\partial t}-v-p \eta \frac{\partial v}{\partial \eta}\right\}=t^{-p} \frac{\partial^{2} v}{\partial \eta^{2}}-\frac{\partial v}{\partial \eta} \text {. }
$$

We now expand

$$
v(\eta, t)=v_{0}(\eta)+o(1)
$$

such that $t(\partial v / \partial t)=o(1)$, as $t \rightarrow \infty, \eta=O(1)$. Substitution into (4.40) gives to leading order

$$
p\left\{v_{0}^{p}+\eta\left(v_{0}^{p}\right)^{\prime}\right\}=v_{0}^{\prime},
$$

where again primes denote differentiation with respect to $\eta$. The general solution is given by

$$
p \eta v_{0}^{p}-v_{0}=C
$$

with $C$ constant. A similar argument to that of section 4.2.1 leads to $C=0$ and the solution for $v_{0}$ is then

$$
v_{0}=(p \eta)_{+}^{1 /(1-p)}
$$

The non-trivial part of the asymptotic solution is confined to the interval $0<\eta<\eta_{2}$ by the mass-invariance condition (4.38), which using (4.41) with (4.42), gives to leading order

$$
M=\int_{0}^{\eta_{2}}(p \eta)^{p /(1-p)} d \eta
$$

Thus

$$
\eta_{2}=\left\{\frac{M}{1-p}\right\}^{1-p} p^{-p}
$$

In terms of $x$ and $t$ and for $0<p<1$ we have now shown that

$$
u(x, t)=t^{-1}\left(\frac{p x}{t^{p}}\right)^{1 /(1-p)}\{1+o(1)\}
$$

as $t \rightarrow \infty, x / t^{p}=O(1)$. The condition $0<\eta<\eta_{2}$ requires that

$$
0<x<\eta_{2} t^{p}
$$

We call (4.44) the outer solution.

We discuss the case $p=1$ and its role in the transition from (4.29) to (4.44) in section 6.

\subsection{The uniformity of the outer solutions: the boundary-layer solutions}

We now consider the question of uniformity in $x$ of the outer solutions for $u(x, t)$ in the limit $t \rightarrow \infty$. In the case $p \geqslant 2$ it is clear that the asymptotic representations (4.16) and (4.30) are uniformly valid for all $x$. For other values of 
$p$ the situation is not so straightforward. For instance when $1<p<2$ we must first ask how (4.25) represents the zero-order asymptotic solution on $-\infty<x<\infty$. Since this has to satisfy $u=0$ at $x= \pm \infty$ we would expect the structure

$$
u(x, t)= \begin{cases}0, & -\infty<x<t+\eta_{1} t^{1 / p}, \\ t^{-1 / p}\left(\frac{t-x}{p t^{1 / p}}\right)^{1 /(p-1)}, & t+\eta_{1} t^{1 / p} \leqslant x<t, \\ 0, & x>t,\end{cases}
$$

There are, however, two objections to this representation. First $u(x, t)$ is not continuous while in the second place, contrary to what we know, the support is bounded. Both these objections to (4.45) can be met by including boundary layers at the trailing and leading edges of the pulse where $\eta=\eta_{1}$ and $\eta=0$ respectively. As $t \rightarrow \infty$ these will be thin on the scale of $\eta$ but since we include the diffusion term within them, they have the effect of smoothing out the solution and at the same time rendering the support unbounded. We note that this approach has been successfully used to uniformize asymptotic solutions to diffusion-convection equations by Grundy $(14,15,16)$.

A similar situation presents itself in the case $0<p<1$ where, in principle, the uniformization can be carried out in the same way. In this case the zero-order outer representation is

$$
u(x, t)= \begin{cases}0, & x<0, \\ t^{-1}\left(\frac{p x}{t^{p}}\right)^{1 /(1-p)}, & 0<x<\eta_{2} t^{p} \\ 0, & x>\eta_{2} t^{p} .\end{cases}
$$

Once again this situation is at variance with what we expect since the solution is discontinuous at $x=\eta_{2} t^{p}$ and there is no moving interface to the left. As in the case $p>1$, these difficulties can be removed by including boundary layers.

Having set out our reasons for seeking boundary-layer solutions we devote the remainder of this section to constructing them.

\subsubsection{The case $1<p<2$.}

(a) The trailing edge layer at $\eta=\eta_{1}$. Near $\eta=\eta_{1}$ we make the change of variable

$$
\eta=\eta_{1}+t^{-\mu} \zeta, \quad \mu>0,
$$

where $\zeta=O(1), t \rightarrow \infty$. This defines a thin layer, on the $\eta$ scale, of thickness $t^{-\mu}$, where $\mu$ has to be found. In this layer we look for a solution which varies on the $\zeta$-scale so we put

$$
v(\eta, t)=w(\zeta, t)
$$


in (4.25) which now becomes

$$
\begin{array}{r}
t^{-\mu}\left\{t \frac{\partial w}{\partial t}-\frac{w}{p}+\left(\mu-\frac{1}{p}\right) \zeta \frac{\partial w}{\partial \zeta}\right\}+t^{-1 / p}\left\{t \frac{\partial\left(w^{p}\right)}{\partial t}+w^{p}+\mu \zeta \frac{\partial\left(w^{p}\right)}{\partial \zeta}\right\}-\frac{\eta_{1}}{p} \frac{\partial w}{\partial \zeta} \\
=t^{\mu-\{(2-p) / p\}} \frac{\partial^{2} w}{\partial \zeta^{2}}+\frac{\partial\left(w^{p}\right)}{\partial \zeta} .
\end{array}
$$

An essential feature of the boundary layer is that the diffusion term becomes important there. Assuming that all $\zeta$-derivatives are bounded within the boundary layer we therefore put

and expand

$$
\mu=\frac{2-p}{p}>0
$$

$$
w(\xi, t)=w_{0}(\xi)+o(1),
$$

with $t(\partial w / \partial t)=o(1)$, as $t \rightarrow \infty, \zeta=O(1)$ in (4.49). Collecting leading-order terms gives

$$
w_{0}^{\prime \prime}+\left(w_{g}^{\prime}\right)^{\prime}+\frac{\eta_{1}}{p} w_{0}^{\prime}=0
$$

with primes denoting differentiation with respect to $\zeta$. The matching condition requires that

$$
w_{0}=\left(\frac{-\eta_{1}}{p}\right)^{1 /(p-1)} \text { as } \zeta \rightarrow \infty
$$

while the boundary conditions are

$$
w_{0}=w_{0}^{\prime}=0 \text { as } \zeta \rightarrow-\infty .
$$

Equation (4.52) admits the solution

$$
w_{0}=\left\{\frac{\eta_{1} e^{-(p-1) \eta_{1}\left(\zeta-\zeta_{0} / p\right.}}{p\left[1-e^{-(p-1) \eta_{1}\left(\zeta-\zeta_{0}\right) / p}\right]}\right\}^{1 /(p-1)}
$$

which satisfies the conditions (4.53) and (4.54) but is only unique to within the arbitrary translational shift $\zeta_{0}$. Unfortunately there appears to be no way of finding $\zeta_{0}$ to this order of approximation but we surmise that this constant will depend on the initial distribution via an integral invariant or through higherorder terms in the asymptotic analysis. However, we have made no attempt to determine its value but it may be possible to fit (4.55) to numerical or experimental data, thereby prescribing a value of $\xi_{0}$.

(b) The leading-edge layer near $\eta=0$. Near $\eta=0$ we make the change of variable

$$
\eta=x t^{-\varepsilon}
$$

where $\varepsilon>0$ will be chosen so that the diffusion term in (4.25) becomes important in the region where the variable $\chi$ is $O(1)$. To see how to scale the 
independent variable $v$ within the trailing edge layer we have the outer expansion

$$
v(\eta, t)=v_{0}(\eta)+o(1)=\left(\frac{-\eta}{p}\right)^{1 /(p-1)}+o(1) \sim t^{-t /(p-1)}\left(\frac{-\chi}{p}\right)^{1 /(p-1)}
$$

in terms of $\chi$. This suggests we put

and expand

$$
v(\eta, t)=t^{-\varepsilon /(p-1)} W(\chi, t)
$$

$$
W(\chi, t)=W_{0}(\chi)+o(1)
$$

with $t(\partial W / \partial t)=o(1)$ as $t \rightarrow \infty, \chi=O(1)$, using

$$
W_{0}(\chi) \sim(-\chi / p)^{1 /(p-1)} \text { as } \chi \rightarrow-\infty
$$

as a matching condition. Making the above changes of variables in (4.25) gives

$$
\begin{aligned}
t \frac{\partial W}{\partial t} & -\left(\frac{\varepsilon}{p-1}+\frac{1}{p}\right) W+\left(\varepsilon-\frac{1}{p}\right) \chi \frac{\partial W}{\partial \chi} \\
+ & t^{-1 / 2}\left\{t \frac{\partial\left(W^{p}\right)}{\partial t}+\left(1-\frac{\varepsilon p}{p-1}\right) W^{p}+\left(\varepsilon-\frac{1}{p}\right) \chi \frac{\partial\left(W^{p}\right)}{\partial \chi}\right\} \\
& =t^{2 t-(2-p) / p} \frac{\partial^{2} W}{\partial \chi^{2}}+\frac{\partial\left(W^{p}\right)}{\partial \chi}
\end{aligned}
$$

We now invoke the condition that the second derivative becomes important in the limit $t \rightarrow \infty, \chi=O(1)$. This demands that

$$
\varepsilon=(2-p) / 2 p
$$

and the equation for $W(\chi, t)$ then becomes

$t \frac{\partial W}{\partial t}-\frac{W}{2(p-1)}-\frac{\chi}{2} \frac{\partial W}{\partial \chi}+t^{-1 / 2}\left\{t \frac{\partial\left(W^{p}\right)}{\partial t}+2 W^{p}-\frac{\chi}{2} \frac{\partial\left(W^{p}\right)}{\partial \chi}\right\}=\frac{\partial^{2} W}{\partial \chi^{2}}+\frac{\partial\left(W^{p}\right)}{\partial \chi}$

Substituting (4.58) into (4.60) and equating leading-order terms gives

$$
W_{0}^{\prime \prime}+\left(W_{\delta}^{p}\right)^{\prime}+\frac{1}{2} \chi W_{0}^{\prime}+\frac{W_{0}}{2(p-1)}=0
$$

with primes indicating $\chi$-derivatives, which has to be solved subject to (4.59) and the boundary conditions

$$
W_{0}=0, \quad \chi \rightarrow \infty .
$$

The boundary-value problem (4.61), (4.59) and (4.62) has been recently studied by Cazenave and Escabedo (17). They show that this problem has a 
family of monotone solutions of which only one has exponential decay of the form $\exp \left(-\chi^{2} / 4\right)$ as $\chi \rightarrow \infty$. The remainder have a power-law decay in the same limit. Based on the non-degenerate nature of equation (4.1) for this range of $p$, we select this unique exponentially decaying solution to the boundary-value problem.

\subsubsection{The case $0<p<1$.}

(a) The leading-edge layer near $\eta=\eta_{2}$. We now go through a similar procedure for the case $0<1<p$, where we expect interfaces to appear within the support of the solution. We put

$$
\eta=\eta_{2}+t^{-\rho} \zeta, \quad \rho>0,
$$

where $\zeta=O(1)$ as $t \rightarrow \infty$, defining a thin layer on the scale of $\eta$ of thickness $t^{-\rho}$, where $\rho$ is to be found. In this layer we look for solutions of (4.35), where

$$
v(x, t)=z(\zeta, t)
$$

which, in the new variables, becomes

$$
\begin{aligned}
t^{-(\mu+1-p)} & \left\{t \frac{\partial z}{\partial t}-z+(\rho-p) \dot{\zeta} \frac{\partial z}{\partial \zeta}\right\}-p \eta_{2} t^{-(1-p)} \frac{\partial z}{\partial \zeta} \\
+ & t^{-\rho}\left\{t \frac{\partial\left(z^{p}\right)}{\partial t}-p z^{p}-(\rho+p) \zeta \frac{\partial\left(z^{p}\right)}{\partial \zeta}\right\}-p \eta_{2} \frac{\partial\left(z^{p}\right)}{\partial \zeta}=t^{\rho-p} \frac{\partial^{2} z}{\partial \zeta^{2}}-\frac{\partial z}{\partial \zeta} .
\end{aligned}
$$

Choosing $\rho=p$ has the effect of making the diffusion term $O(1)$ so the equation for $z(\zeta, t)$ becomes

$$
\begin{array}{r}
t^{-1}\left\{t \frac{\partial z}{\partial t}-z\right\}-p \eta_{2} t^{-(1-p)} \frac{\partial z}{\partial \zeta}+t^{-p}\left\{t \frac{\partial\left(z^{p}\right)}{\partial t}-p z^{p}-2 p \zeta \frac{\partial\left(z^{p}\right)}{\partial \zeta}\right\} \\
=\frac{\partial^{2} z}{\partial \zeta^{2}}-\frac{\partial z}{\partial \zeta}+p \eta_{2} \frac{\partial\left(z^{p}\right)}{\partial \zeta} .
\end{array}
$$

Expanding

$$
z(\zeta, t)=z_{0}(\zeta)+o(1)
$$

such that $t(\partial z / \partial t)=o(1)$ as $t \rightarrow \infty, \zeta=O(1)$, yields the equation

$$
z_{0}^{\prime \prime}-z_{0}^{\prime \prime}+p \eta_{2}\left(z_{0}^{p}\right)^{\prime}=0
$$

for $z_{0}$, with primes denoting differentiation with respect to $\zeta$. This has to be solved subject to the matching condition

$$
z_{0} \rightarrow\left(p \eta_{2}\right)^{1 /(1-p)}, \quad \zeta \rightarrow-\infty
$$

and the boundary condition

$$
z_{0}=z_{0}^{\prime}=0, \quad \zeta \rightarrow \infty .
$$


The problem (4.65) to (4.67) admits the solution

$$
z_{0}= \begin{cases}{\left[p \eta_{2}-e^{(1-p)\left(\zeta-\zeta_{0}\right)}\right]^{1 /(1-p)},} & \zeta<\zeta_{0}+\frac{\log \left(p \eta_{2}\right)}{1-p} \\ 0, & \zeta>\zeta_{0}+\frac{\log \left(p \eta_{2}\right)}{1-p}\end{cases}
$$

which is unique to within the arbitrary shift $\zeta_{0}$ which is not determined to this order of approximation. Clearly (4.68) has an interface at

$$
\xi=\zeta_{0}+\frac{\log \left(p \eta_{2}\right)}{(1-p)} .
$$

(b) The trailing-edge layer near $\eta=0$. In order to work out the structure of the asymptotic solution near $\eta=0$ we have to look in a little more detail at the outer expansion (4.41). To be specific we write

$$
v(\eta, t)=(p \eta)^{1 /(1-p)}+t^{-p} \log t v_{1}(\eta)+t^{-p} v_{2}(\eta)+t^{-(1-p)} v_{3}(\eta)+\cdots
$$

Substituting this into (4.40) we find that

and

$$
\begin{aligned}
& v_{1}=K(p \eta)^{p /(1-p)} \\
& v_{2}=\frac{(p \eta)^{p /(1-p)}}{(1-p)} \log \eta\left\{\frac{p^{2}}{(1-p)^{2}}-K\right\}+C_{2}(\eta p)^{p /(1-p)}
\end{aligned}
$$

$$
v_{3}=\frac{(\eta p)^{(2-p) /(1-p)}}{p(1-p)}+C_{3} \eta p,
$$

where $K, C_{2}$ and $C_{3}$ are arbitrary constants. Comparing the first two terms in (4.70) with (4.71) reveals that the outer expansion is non-uniformly valid when $x=O(\log t)$, suggesting the inner independent variable

$$
\zeta=\frac{x}{\log t}=\frac{\eta t^{p}}{\log t}
$$

together with the dependent variable

$$
u=\left(\frac{\log t}{t}\right)^{1 /(1-p)} Z(\zeta, t)
$$

where we have reverted to the original independent variable $u$. Making these changes of variables in (4.1) and expanding

yields the equation

$$
Z(\zeta, t)=Z_{0}(\zeta)+o(1)
$$

$$
Z_{0}^{\prime}=\frac{p}{(1-p)} Z_{0}
$$


with solution

$$
Z_{0}=\left\{p\left(\zeta-\zeta_{0}\right)\right\}^{1 /(1-p)}
$$

giving an interface at $\zeta=\zeta_{0}$ which is, at the moment, unknown.

To match the inner and outer expansions we first recast (4.70) with (4.71) to (4.73) in terms of $\zeta$ and $u$ to give

$$
u=\left(\frac{\log t}{t}\right)^{1 /(1-p)}\left\{(p \zeta)^{1 /(1-p)}+\frac{(p \zeta)^{p /(1-p)}}{(1-p)}\left[K-\frac{p^{2}}{(1-p)^{2}}\right]+O\left(\frac{\log (\log t)}{\log t}\right)\right\}
$$

This must match with (4.71) and (4.73) expanded as $\zeta \rightarrow \infty$, namely

$$
u=\left(\frac{\log t}{t}\right)^{1 /(1-p)}\left\{(p \zeta)^{1 /(1-p)}-\frac{p \zeta_{0}}{(1-p)}(p \zeta)^{p /(1-p)}+\ldots\right\}
$$

Thus for a successful match we require that

$$
p \zeta_{0}=\frac{p^{3}}{(1-p)^{2}}-K
$$

To proceed further we note that the diffusion term has yet to be taken into account in the inner region. In order to do this we make a further scaling near $\zeta=\zeta_{0}$, namely

$$
\zeta=\zeta_{0}+\frac{\xi}{\log t}
$$

together with

$$
u=t^{-1 /(1-p)} U(\xi, t)
$$

We observe in passing that in terms of $x$ the scaling (4.80) can be written as

$$
x=\zeta_{0} \log t+\xi .
$$

Making these changes of variables the equation for $U(\xi, t)$ becomes

$$
t^{-1}\left\{t \frac{\partial U}{\partial t}-\frac{U}{(1-p)}-\zeta_{0} \frac{\partial U}{\partial \xi}\right\}+t \frac{\partial\left(U^{p}\right)}{\partial t}-\frac{p}{(1-p)} U^{p}+\zeta_{0} \frac{\partial\left(U^{p}\right)}{\partial \xi}=\frac{\partial^{2} U}{\partial \xi^{2}}-\frac{\partial U}{\partial \xi} .
$$

Expanding

$$
U(\xi, t)=U_{0}(\xi)+o(1)
$$

such that $t(\partial U / \partial t)=o(1)$ as $t \rightarrow \infty, \xi=O(1)$ gives the ordinary differential equation

$$
U_{0}^{\prime \prime}-U_{0}^{\prime}+\frac{p}{(1-p)} U_{b}+\zeta_{0}\left(U_{0}\right)^{\prime}=0
$$

for $U_{0}$ with primes denoting differentiation with respect to $\xi$. The matching 
condition with the inner solution requires that

$$
U_{0} \sim(p \xi)^{1 /(1-p)}, \quad \xi \rightarrow \infty
$$

while the boundary condition demands that

$$
U_{0}=0, \quad \xi \rightarrow-\infty .
$$

The problem is to find $\zeta_{0}$ and $U_{0}$ subject to (4.84) to (4.86).

We show in Appendix B that a unique solution to this problem exists if and only if

and in that event

$$
\zeta_{0}=-p /(1-p)
$$

$$
U_{0}= \begin{cases}{\left[p\left(\xi-\xi_{1}\right)\right]^{1 /(1-p)},} & \xi \geqslant \xi_{1}, \\ 0, & \xi<\xi_{1}\end{cases}
$$

for some $\xi_{1}$. So, returning to (4.83) we find that

$$
K=p^{2} /(1-p)^{2} \text {. }
$$

We note that (4.88) is actually uniformly valid throughout the inner region and can be matched to zero order with the outer expansion. We use this observation in the next section, where we construct uniformly valid solutions for all $x$.

Finally from (4.88) we observe that the trailing-edge interface is located at $\xi=\xi_{1}$ or in view of (4.87) and (4.82) at

$$
x=\frac{-p}{(1-p)} \log t+\xi_{1} .
$$

\subsection{Construction of uniformly-valid solution for $0<p<2$}

In this section we make some remarks about the construction of uniformlyvalid large-time solutions for the parameter range $0<p<2$. In contrast to the situation for $p \geqslant 2$, for $0<p<2$ the asymptotic analysis does not yield a single formula which approximates $u(x, t)$ uniformly in $x$ as $t \rightarrow \infty$. To see how such a formula may be constructed we note that the asymptotic solution for both $0<p<1$ and $1<p<2$ can be regarded as an outer expansion linked by two boundary layers to the conditions at infinity. Although this picture is not entirely straightforward for $0<p<1$ the interpretation can still be made since, as noted above, equation (4.88) is uniformly valid in the trailing-edge layer.

If we denote the zero-order solution by $U_{0}$, the zero-order leading-edge boundary-layer solution by $U_{1}$ and the zero-order trailing-edge boundary-layer solution by $U_{2}$ then for $0<p<1$ we have from (4.46)

$$
U_{0}= \begin{cases}0, & x<0, \\ \left(\frac{p x}{t}\right)^{1 /(1-p)}, & 0<x \leqslant \eta_{2} t^{p}, \\ 0, & x>\eta_{2} t^{p},\end{cases}
$$


and from (4.88)

$$
U_{1}= \begin{cases}t^{-1}\left[p \eta_{2}-e^{(1-p)\left(x-\eta_{2} t^{\prime}-\zeta_{0}\right)}\right]^{1 /(1-p)}, & x<\eta_{2} t^{p}+\zeta_{0}+\frac{\log \left(p \eta_{2}\right)}{1-p}, \\ 0, & x>\eta_{2} t^{p}+\zeta_{0}+\frac{\log \left(p \eta_{2}\right)}{1-p},\end{cases}
$$

while from (4.88) with (4.82)

$$
U_{2}= \begin{cases}0, & x<\xi_{1}-\frac{p}{1-p} \log t, \\ t^{-1 /(1-p)}\left\{p\left(x+\frac{p}{1-p} \log t-\xi_{1}\right)\right\}^{1 /(1-p)}, & x \geqslant \xi_{1}-\beta \log t .\end{cases}
$$

For $1<p<2$, the corresponding expressions and domains are from (4.45)

from (4.55)

$$
U_{0}= \begin{cases}0, & x<t+\eta_{1} t^{1 / p} \\ t^{-1 /(p-1)}\left(\frac{t-x}{p}\right)^{1 /(p-1)}, & t+\eta_{1} t^{1 / p} \leqslant x<t \\ 0, & x>t\end{cases}
$$

$$
U_{1}=t^{-1 / p}\left\{\frac{\eta_{1}}{p\left(\exp \left\{(p-1) \eta_{1}\left(x-t-\eta_{1} t^{1 / p}-\zeta_{0} t^{(p-1) / p}\right) / p t^{(p-1) / p}\right\}-1\right)}\right\}^{1 /(p-1)},
$$

and from (4.58)

$$
-\infty<x<\infty
$$

$$
U_{2}=t^{-1 / 2(p-1)} W_{0}\left(\frac{x-t}{t^{1 / 2}}\right), \quad-\infty<x<\infty .
$$

The next step in the process is to construct the inner limits of the outer solution, expressions which have to reflect the failure of analyticity of $U_{0}$ at the leading and trailing edges. For $0<p<1$ we write the leading-edge inner limit of $U_{0}$ as

$$
\left(U_{0}\right)_{1}= \begin{cases}t^{-1}\left(p \eta_{2}\right)^{1 /(1-p)}, & x \leqslant \eta_{2} t^{p} \\ 0, & x>\eta_{2} t^{p}\end{cases}
$$

and the trailing-edge inner limit of $U_{0}$ as

$$
\left(U_{0}\right)_{2}= \begin{cases}t^{-1 /(1-p)}(p x)^{1 /(1-p)}, & x>0 \\ 0, & x<0 .\end{cases}
$$

For $1<p<2$, corresponding expressions are

$$
\left(U_{0}\right)_{1}= \begin{cases}0, & x<t+\eta_{1} t^{1 / p} \\ t^{-1 / p}\left(-\eta_{1} / p\right), & x \geqslant t+\eta_{1} t^{1 / p}\end{cases}
$$


and

$$
\left(U_{0}\right)_{2}= \begin{cases}t^{-1 /(p-1)}\left(\frac{t-x}{p}\right)^{1 /(p-1)}, & x<t, \\ 0, & x \geqslant t .\end{cases}
$$

The idea now is to construct what, in the language of matched expansions, are called composite solutions and which are uniformly valid for all $x$. In this case we use multiplicative composition; for a number of reasons the more familiar additive composition is not applicable here. In a more straightforward situation a leading-order composite approximation would be of the form

$$
u_{c}(x, t)=\frac{U_{0} U_{1} U_{2}}{\left(U_{0}\right)_{1}\left(U_{0}\right)_{2}}
$$

(see for example van Dyke (18)). However this rule cannot be applied directly since $U_{0},\left(U_{0}\right)_{1}$ and $\left(U_{0}\right)_{2}$ are zero over certain domains of $x$. Nevertheless the multiplicative nature of $u_{c}$ makes it a straightforward matter to modify (4.89) to take this into account. In the case $0<p<1$ we write

$$
u_{c}(x, t)= \begin{cases}0, & x<\xi_{1}-\log t \\ \frac{U_{1} U_{2}}{\left(U_{0}\right)_{1}}, & \xi_{1}-\log t<x<0 \\ \frac{U_{1} U_{2}}{\left(U_{0}\right)_{1}}, & 0<x<\eta_{2} t^{p} \\ \frac{U_{1} U_{2}}{\left(U_{0}\right)_{2}}, & \eta_{1} t^{p}<x<\eta_{2} t^{p}+\xi_{0}+\frac{\log \left(p \eta_{2}\right)}{1-p} \\ 0, & x>\eta_{2} t^{p}+\xi_{0}+\frac{\log \left(p \eta_{2}\right)}{1-p}\end{cases}
$$

This is adapted from (4.89) by omitting $U_{0}=\left(U_{0}\right)_{2}=0$ from both numerator and denominator in the second domain. In the third domain $U_{0}=\left(U_{0}\right)_{2}$ which cancel while in the fourth domain $U_{0}=\left(U_{0}\right)_{1}=0$ which can again be omitted. The corresponding result for $1<p<2$ is

$$
u_{c}(x, t)= \begin{cases}\frac{U_{1} U_{2}}{\left(U_{0}\right)_{2}}, & x<t+\eta_{2} t^{1 / p}, \\ \frac{U_{1} U_{2}}{\left(U_{0}\right)_{1}}, & t+\eta_{1} t^{1 / p}<x<t, \\ \frac{U_{1} U_{2}}{\left(U_{0}\right)_{1}}, & x>t .\end{cases}
$$

Although these uniformly valid composite expansions are continuous, they accept 
discontinuities in their $x$-derivatives; namely of $O\left(t^{-(p+1)}\right)$ at $x=\eta_{2} t^{p}$ for $0<p<1$ and of $O\left(t^{-2 / p}\right)$ at $x=t+\eta_{1} t^{1 / p}$ for $1<p<2$. However, this is not a high price to pay since in each case the formula for $u_{c}$ is simple and in the case $0<p<1$ very easy to compute. For $1<p<2$ the procedure is complicated by the need to evaluate $W_{0}\left((x-t) / t^{1 / 2}\right)$ at prescribed values of its argument.

The main problem with using the composite solutions is that they are indeterminate to within the two arbitrary constants occurring in each of the boundarylayer solutions. In principle these cannot be determined from the zero-order analysis. However, it is possible to simulate the convergence to the asymptotic solution by adjusting these constants so that the composite solution fits, in some ad hoc way, the numerical solution at a suitable value of time. From a practical computational point of view the use of such a formula would reduce the excessive computing time required to calculate the slowly converging large-time solution using the scheme of section 5 .

\section{The numerical method}

The method used to generate the numerical solutions was developed and analysed by Dawson (19) for nonlinear parabolic equations in one space dimension. Here, we discuss the application of the method to the problem (2.11), (2.12).

Again we make transformation (3.3) and use the transformed equation as a starting point for the discussion. However, here we want to keep the diffusion coefficient $D$ in the analysis, because this will allow us to do computations for problems in which convection dominates diffusion. Therefore we consider, with $w=\beta(u)$ and $u=\varphi(w)$,

$$
\frac{\partial w}{\partial t}+\frac{\partial \varphi(w)}{\partial x}-D \frac{\partial^{2} \varphi(w)}{\partial x^{2}}=0, \quad(x, t) \in Q .
$$

Let $-\infty<\cdots<x_{-J-1 / 2}<x_{-J+1 / 2}<\infty$ be a partition of $\mathbb{R}$ into grid blocks $B_{j}=\left[x_{j-1 / 2}, x_{j+1 / 2}\right]$, and let $x_{j}$ be the midpoint of $B_{j}$. For simplicity, assume that the partition is uniform with mesh spacing $h>0$. Let $\Delta t>0$ denote a time-stepping parameter, and let $t^{n}=n \Delta t$ and $t^{n+1 / 2}=\left(t^{n}+t^{n+1}\right) / 2$. For functions $g(x, t)$, let $g_{j}^{n}=g\left(x_{j}, t^{n}\right), g_{j+1 / 2}^{n+1 / 2}=g\left(x_{j+1 / 2}, t^{n+1 / 2}\right)$, etc.

On each grid block $B_{j}$, approximate $w_{j}^{n}$ and $u_{j}^{n}$ by constants $\omega_{j}^{n}$ and $U_{j}^{n}$ respectively, where

$$
\omega_{j}^{n} \equiv U_{j}^{n}+\left(U_{j}^{n}\right)^{p}
$$

Discretizing (5.1) by finite differences in space and time, we find that

$$
\frac{w_{j}^{n+1}-w_{j}^{n}}{\Delta t}+\frac{\varphi\left(w_{j+1 / 2}^{n+1 / 2}\right)-\varphi\left(w_{j-1 / 2}^{n+1 / 2}\right)}{h}-D \frac{\varphi\left(w_{j+1}^{n+1}\right)-2 \varphi\left(w_{j}^{n+1}\right)+\varphi\left(w_{j-1}^{n+1}\right)}{h^{2}} \approx 0 \text {. }
$$

The term $\varphi\left(w_{j+1 / 2}^{n+1 / 2}\right)$ is approximated using a higher-order Godunov approach, 
see van Leer (20). Assume the time step satisfies the CFL constraint

$$
\sup _{w} \varphi^{\prime}(w) \Delta t \leqslant h \text {. }
$$

Expanding in a Taylor series about the point $\left(x_{j}, t^{n}\right)$,

$$
w_{j+1}^{n+1 / 2}=w+\frac{h}{2} w_{x}+\frac{\Delta t}{2} w_{t}+O\left(h^{2}+\Delta t h+\Delta t^{2}\right),
$$

where the right-hand side of $(5.5)$ is evaluated at $\left(x_{j}, t^{n}\right)$. Using the differential equation $(5.1)$, we find that

$$
w_{j+1 / 2}^{n+1 / 2}=w+\left(\frac{h}{2}-\varphi^{\prime}(w) \frac{\Delta t}{2}\right) w_{x}+O\left(h^{2}+\Delta t\right)
$$

Emulating (5.6), define

$$
\bar{\omega}_{j+1 / 2}^{n+1 / 2}=\omega_{j}^{n}+\left(\frac{h}{2}-\varphi^{\prime}(\omega)_{j}^{n}\right) \frac{\Delta t}{2} \delta_{x} \omega_{j}^{n}
$$

where $\delta_{x} \omega_{j}^{n}$ is calculated by a slope-limiting procedure. In particular

where

$$
\delta_{x} \omega_{j}^{n}=\delta_{\lim } \omega_{j}^{n} \cdot \operatorname{sign}\left(\omega_{j+1}^{n}-\omega_{j-1}^{n}\right),
$$

$$
\delta_{\lim } \omega_{j}^{n}= \begin{cases}\min \left(\left|\Delta_{+} \omega_{j}^{n}\right|,\left|\Delta_{-} \omega_{j}^{n}\right|\right) & \text { if } \Delta_{+} \omega_{j}^{n} \cdot \Delta_{-} \omega_{j}^{n}>0, \\ 0 & \text { otherwise. }\end{cases}
$$

Here $\Delta_{+} \omega_{j}$ is the forward difference $\left(\omega_{j+1}-\omega_{j}\right) / h$, and $\Delta_{-} \omega_{j}=\left(\omega_{j}-\omega_{j-1}\right) / h$. Approximating $\omega_{j+1}^{n-1} / 2$ by $\bar{\omega}_{j+1 / 2}^{n+1 / 2}$, we obtain the nonlinear system of equations

$$
\begin{aligned}
\frac{\omega_{j}^{n+1}-\omega_{j}^{n}}{\Delta t} & +\frac{\varphi\left(\bar{\omega}_{j+1}^{n+1 / 2}\right)-\varphi\left(\bar{\omega}_{j-1}^{n+1 / 2}\right)}{h} \\
& -D \frac{\varphi\left(\omega_{j+1}^{n+1}\right)-2 \varphi\left(\omega_{j}^{n+1}\right)+\varphi\left(\omega_{j-1}^{n+1}\right)}{h^{2}}=0 .
\end{aligned}
$$

Initially, set $U_{j}^{0}=u^{0}\left(x_{j}\right)$ and $\omega^{0}=U_{j}^{0}+\left(U_{j}^{0}\right)^{p}$. Note that, given $\omega_{j}^{n}$ at some time level $t^{n}$, the term $\varphi\left(\bar{\omega}_{j+1 / 2}^{n+1 / 2}\right)$ is calculated explicitly. Thus, we are left with a symmetric system of nonlinear equations to determine $\omega_{j}^{n+1}$, which we solve by a fixed-point iteration.

Let

$$
r_{j}^{n}=-\frac{\varphi\left(\bar{\omega}_{j-1}^{n+1 / 2}\right)-\varphi\left(\bar{\omega}_{j-1 / 2}^{n+1 / 2}\right)}{h} .
$$

Then, substituting the definition of $\omega_{j}^{n+1}$ into (5.10) we find that

$$
\frac{U_{j}^{n+1}-U_{j}^{n}}{\Delta t}+\frac{\left(U_{j}^{n+1}\right)^{p}-\left(U_{j}^{n}\right)^{p}}{\Delta t}-D \frac{U_{j+1}^{n+1}-2 U_{j}^{n+1}+U_{j-1}^{n+1}}{h^{2}}=r_{j}^{n} \text {. }
$$


Let $U_{j}^{n+1,0}$ be an initial guess for $U_{j}^{n+1}$. Then, given $U_{j}^{n+1, k}$, define

$$
\beta_{j}^{n, k}= \begin{cases}\frac{\left(U_{j}^{n+1, k}\right)^{p}-\left(U_{j}^{n}\right)^{p}}{U_{j}^{n+1, k}-U_{j}^{n}}, & \text { if } U_{j}^{n+1, k} \neq U_{j}^{n}, \\ 0, & \text { otherwise }\end{cases}
$$

and determine the $(k+1)$ th iterate $U_{j}^{n+1, k+1}$

$$
\left[1+\beta_{j}^{n, k}\right] \frac{U_{j}^{n+1, k+1}-U_{j}^{n}}{\Delta t}-D \frac{U_{j+1}^{n+1, k+1}-2 U_{j}^{n+1, k+1}+U_{j-1}^{n+1, k+1}}{h^{2}}=r_{j}^{n} \text {. }
$$

This procedure gives iterates which satisfy a maximum principle; that is

$$
\min _{j} U_{j}^{n} \leqslant U_{j}^{n+1, k} \leqslant \max _{j} U_{j}^{n}
$$

therefore $\beta_{j}^{n, k}$ well defined.

\section{Summary and discussion}

By way of conclusion we summarize our results. For $p>2$ the limit profile is given by the leading term in (4.16). This is a symmetric profile which travels to the right with unit speed decaying like $t^{-1 / 2}$. Numerical solutions of the initial-value problem for (2.11) with $p=3$ and $D=1$ are displayed in Figs 1 and 2 with $t^{1 / 2} u$ as a function of $\eta=(x-t) / t^{1 / 2}$ for various values of $t$. These clearly confirm the analytic behaviour.

For $p=2$ the diffusion-dominated profile becomes asymmetric due to the influence of convection. The limiting solution is in fact a similarity solution of Burgers's equation. Numerical convergence to the limit profile is illustrated in Fig. 3.

For $1<p<2$ the asymptotic profile still moves to the right with unit speed. It is left-asymmetric with respect to $x=t$ and has a discontinuity along the curve $(x-t) / t^{1 / p}=\eta_{1}$, with $\eta_{1}$ given by (4.29). In section 4.2 boundary-layer solutions are constructed near the leading and trailing edges of the outer solution which resolve its non-analytic behaviour near $\eta=0$ and $\eta=\eta_{1}$. Figure 4 presents sample numerical solutions for the case $p=\frac{3}{2}$. The main aim here is to show convergence to the outer solution. In order to reduce the spatial effect of the boundary layers, which have thickness proportional to $D^{\alpha} t^{-\beta}$ for some $\alpha, \beta>0$, we take $D=10^{-2}$. To check the convergence properties of this numerical solution we compare the computations with the analytic solution of the initial value problem for $D=0$. The solution is written down for $p=\frac{3}{2}$ in Appendix $\mathrm{C}$ and presented in Fig. 5. The slow convergence to the outer limit, shared by both sets of results, is clearly evident.

As $p \downarrow 1$ the outer expansion is no longer valid. This is clear from (4.25). However, we can solve the asymptotic problem for $p=1$ (see (3.15)) to give

$$
u(x, t) \sim \frac{M}{(2 \pi t)^{\frac{1}{t}}} \exp \left\{-\frac{(\mathrm{x}-t / 2)^{2}}{2 t}\right\}, \quad t \rightarrow \infty,
$$




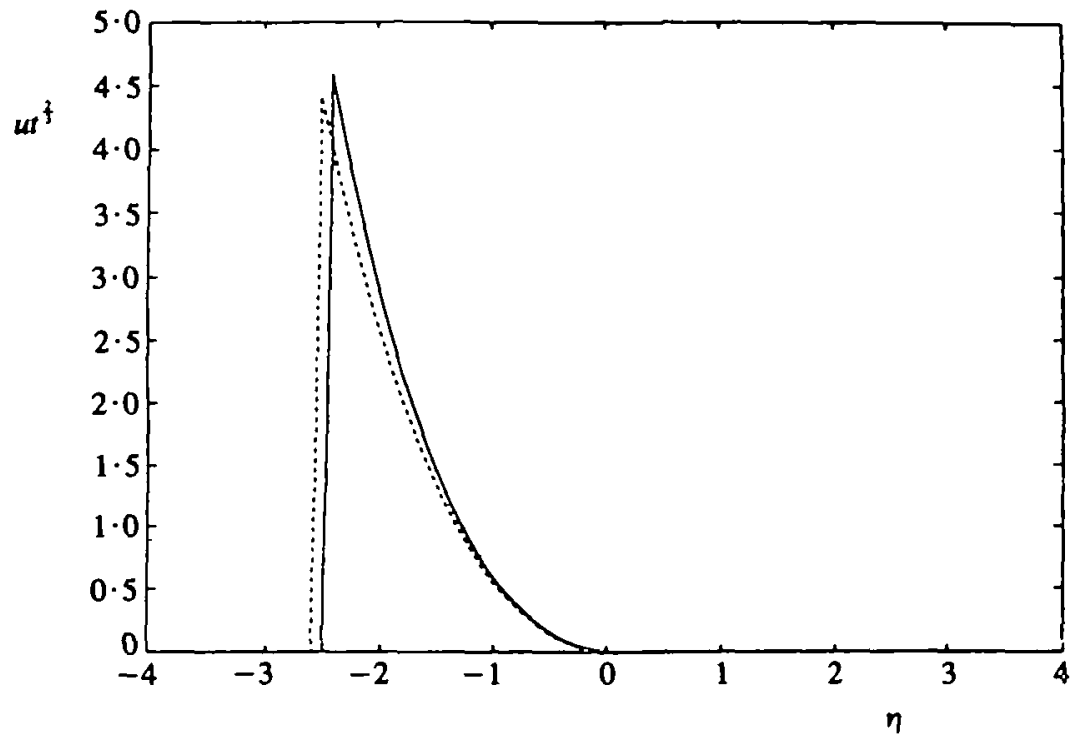

FIG. 4. Convergence of the numerical solution to the zero- order outer solution for $p=1 \cdot 5$. $u t^{2 / 3}$ plotted as a function of $\eta$ for $-t=1000, \cdots t=2000$ with $D=10^{-2}$

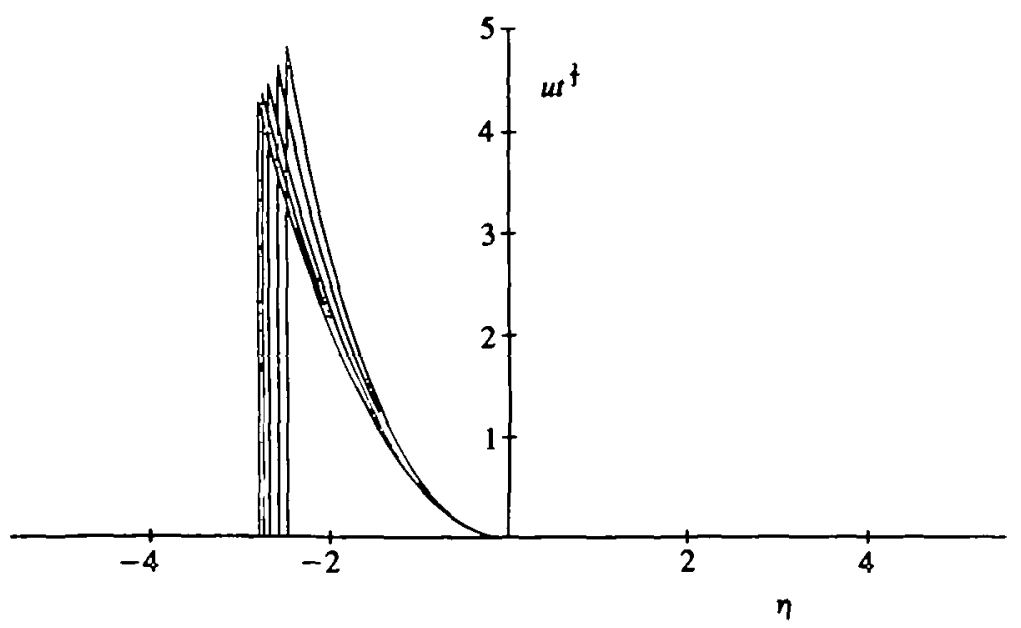

FIG. 5. Convergence of the exact solution of the initial-value problem to the zero-order outer solution for $D=0$ and $p=1 \cdot 5$. The values of time are 1000 , $2000,5000,10000$ and 20000

a diffusion-dominated profile moving with speed $\frac{1}{2}$ to the right. Bearing in mind the change in translation properties of the solution as $p$ passes through 1 , it is not surprising that the limiting form for $0<p<1$ does not translate at all. In this regime the relevant outer variable is $\eta=x / t^{p}$ and the outer solution 


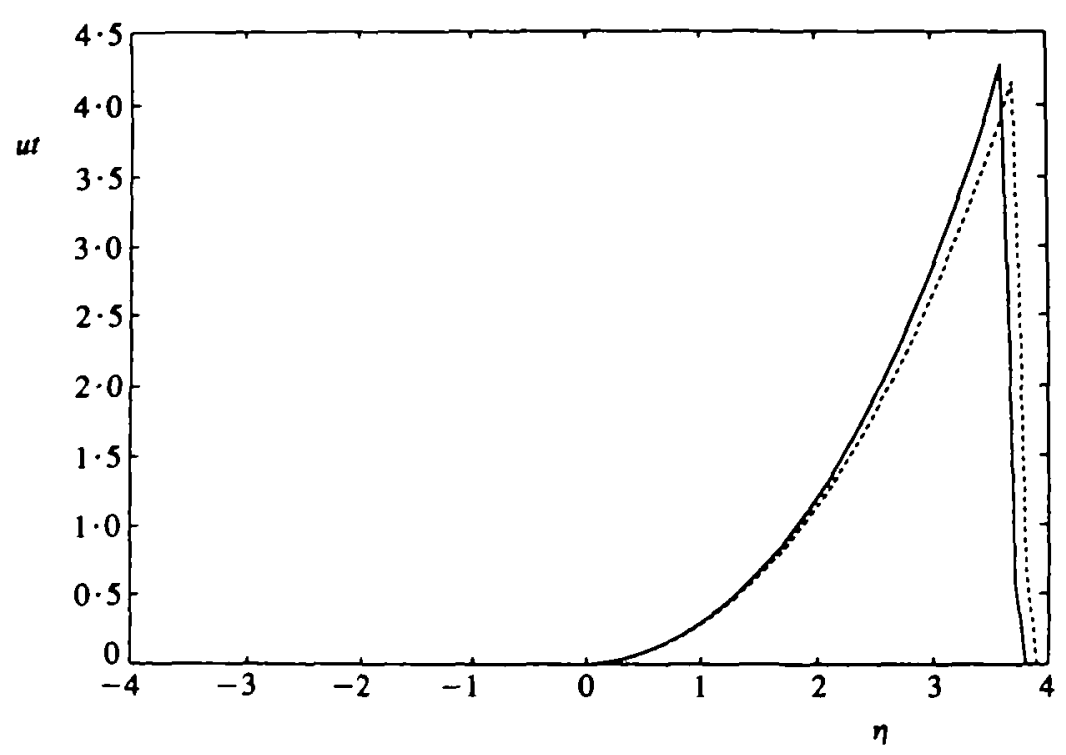

FIG. 6. Convergence of the numerical solution to the zero-order outer solution for $p=0-5$. ut plotted as a function of $\eta$ for $-t=1000,--t=2000$ with $D=10^{-2}$

becomes right symmetric with respect to $x=0$ with a discontinuity along the curve $x=\eta_{2} t^{p}$, with $\eta_{2}$ determined by (4.43). Again we can construct boundarylayer solutions near $\eta=0$ and $\eta=\eta_{2}$, which in both cases lead to solvable problems predicting interfaces. Figure 6 shows results of the computations for the initial-value problem (2.11) with $D=10^{-2}$ to isolate the outer solution from the influence of the boundary layers. As a check on the numerical convergence we again compare the computations with the analytic solution for $D=0$ given in Appendix $C$ and displayed in Fig. 7. In both cases the slow convergence to the outer solution is apparent.

The results of the paper clearly distinguish the difference between the cases $p>1$ and $p<1$. This can also be interpreted in terms of the underlying physical model; see also van Genuchten and Cleary (6), in the following way.

The differential capacity for adsorption, which is obtained from equation (1.1) by differentiating the nonlinear term in the time derivative, that is,

$$
p u^{p-1}+1
$$

shows that for $p>1$ the adsorption at low concentration is relatively minor. Consequently the leading edge of the profile propagates at a speed nearly equal to that of the fluid. This is consistent with the translational properties of the outer solution as given by (4.1) for $p>1$. At higher concentrations adsorption becomes much more extensive, resulting in a lower speed of propagation. This 


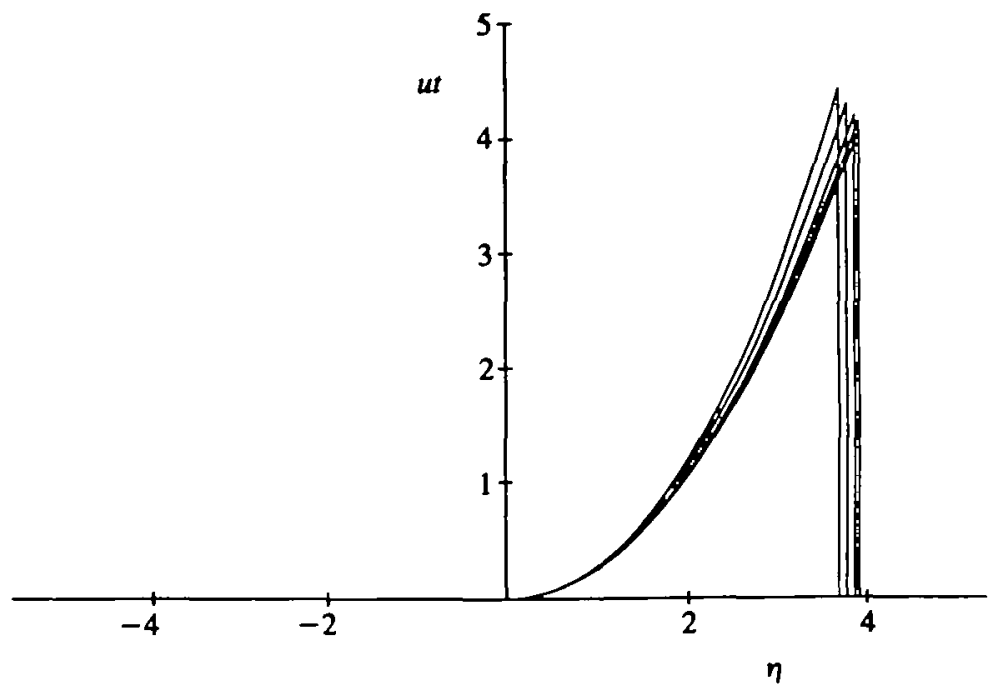

FIG. 7. Convergence of the exact solution to the initial value problem for $D=0$ and $p=0.5$. The values of time are $1000,2000,5000,10000$ and 20000

will cause the profile to be flat or dispersed behind the leading edge. After most of the chemical has passed and the concentration starts decreasing, much of the chemical which was adsorbed will go back into solution (capacity relatively large). Consequently the concentration tends to remain high, leading to a sharp front at the trailing edge of the profile. Again this agrees with the representation (4.45) and the computations shown in Fig. 4. As $p$ increases the effect of absorption becomes progressively weaker and the transport of the solute will be governed by diffusion. Our analyses show that $p=2$ is asymptotically a critical value in this respect since for $p>2$ the limiting profile is dominated by diffusion. See Figs 1, 2 and 3.

When $p=2$, the differential capacity is constant and equal to 2 . Therefore all concentrations move with half the speed of the fluid, leading to a symmetrical profile of the form (3.16).

Finally, $0<p<1$. Here the capacity decreases with increasing concentration. This means that as concentration increases, the resulting adsorption rate becomes smaller, leading to an increase in propagation speed. Consequently the solute distribution becomes steep at the leading edge of the profile. As concentration decreases, the desorption rate of the chemical increases, bringing more and more chemical into solution and hence reducing the decrease in the solution concentration. This leads to a highly dispersed solute profile near the trailing edge. This physical interpretation is supported by both the limiting solution (4.44) and the computations presented in Fig. 6. 


\section{Acknowledgements}

The first two authors acknowledge the support of EC contract SC1-019C(TT). The authors would like to thank Rund Schotting for computational assistance during the preparation of this paper.

\section{REFERENCES}

1. M. Escabedo, J. L. VAzQueZ and E. ZuAzuA. Source type solutions and asymptotic behaviour for a diffusion convection equation, preprint.

2. J. Bear, Dynamics of Fluids in Porous Media, 2nd edition (Elsevier, New York 1972).

3. R. A. Freeze and J. A. Cherry, Groundwater (Prentice-Hall, Englewood Cliffs 1979).

4. G. H. Bolt, Soil Chemistry B. Physico-Chemical Models (Elsevier, Amsterdam 1979).

5. C. J. van DUiJn and P. KNABNeR, Transport in Porous Media 8 (1992) 167-226.

6. M. Th. van GenuChten and R. W. Cleary, In Soil Chemistry B, Physico-Chemical Models (ed. G. H. Bolt; Elsevier, Amsterdam 1982) 349-386.

7. P. KNabner, Meth. Verf. math. Phys. 36 (1991).

8. C. J. VAN DUIJN and P. KNABNER, J. Reine angew. Math. 415 (1991) 1-49.

9. —— and ibid. 18 (1987) 728-743.

10. B. H. Gilding, Ann. Scuola, Norm. Sup. Pisa Cl. Sci. 16 (1989) 165-224.

11. S. Osher and J. Ralston, Comm. pure appl. Math. 35 (1982) 737-749.

12 C. J. van Duinn and J. M. DE GraAf, SI AM J. math. Anal. 18 (1987) 728-743.

13. M. Escabedo and E. Zuazua, J. Funct. Anal. 100 (1991) 119-161.

14. R. E. GRUNDy, IMA J. appl. Math. 31 (1983) 121-137.

15. — ibid. 40 (1988) 183-204.

16. — ibid. 40 (1988) 53-72.

17. T. CAZENAVE and M. ESCABEDO, A two-parameter shooting problem for a secondorder differential equation, preprint.

18. M. VAN DYKE, Perturbation Methods in Fluid Mechanics (Academic Press, New York 1964).

19. C. N. Dawson, SIAM J. numer. Anal. to appear.

20. B. VAN LeER, J. comput. Phys. 32 (1979) 101-136.

\section{APPENDIX A}

Error analysis for $p=3$ and $p=2$

In the first part of this Appendix we compute the leading error term in the expansion (4.14) for $v(\eta, t)$ when $p=3$. It turns out that we must consider an expansion of the form

$$
v(\eta, t)=v_{0}(\eta)+t^{-1 / 2}(\log t) v_{1}(\eta)+t^{-1 / 2} v_{2}(\eta)+o\left(t^{-1 / 2}\right),
$$

where

$$
v_{0}(\eta)=\frac{M}{2 \sqrt{ } \pi} e^{-\pi^{2} / 4} .
$$

With a few modifications the analysis can be extended to all values of $p>2$. Substituting (A.1) into (4.13) and equating terms of $O\left(t^{-1 / 2} \log t\right)$ gives

$$
v_{1}^{\prime \prime}+\frac{1}{2} \eta v_{1}^{\prime}+v_{1}=0, \quad v_{1}( \pm \infty)=0
$$

If we require $v_{1}$ to have exponential decay as $\eta \rightarrow \pm \infty$, then (A.2) has the solution

$$
v_{1}=A v_{0}^{\prime}(\eta)
$$


where $A$ is an arbitrary constant. The equation for $v_{2}$ can now be written as

with solution

$$
v_{2}^{\prime \prime}+\frac{1}{2} \eta v_{2}^{\prime}+v_{2}=\left(A-3 v_{0}^{2}\right) v_{0}^{\prime}
$$

where

$$
v_{2}=v_{0}^{\prime}(\eta) \omega_{2}(\eta)
$$

$$
\omega_{2}^{\prime}=\frac{B e^{\eta^{2} / 4}}{\eta^{2}}+\frac{3 M^{2}}{4 \pi} \frac{e^{\eta^{2} / 4}}{\eta^{2}} \int_{\eta}^{\infty} s^{2} e^{-3 s^{2} / 4} d s-\frac{A e^{\eta^{2} / 4}}{\eta^{2}} \int_{\pi}^{\infty} s^{2} e^{-s^{2} / 4} d s
$$

and $B$ is an arbitrary constant. We now require that $v_{2}$ decays exponentially to zero as $\eta \rightarrow \pm \infty$. This demands that the exponentially growing terms in $\omega_{2}^{\prime}$ be suppressed, which, as $\eta \rightarrow+\infty$, requires $B$ to be zero. The same condition as $\eta \rightarrow-\infty$ gives

$$
A=\frac{3 M^{2}}{4 \pi} \int_{-\infty}^{\infty} s^{2} e^{-3 s^{2} / 4} d s / \int_{-\infty}^{\infty} s^{2} e^{-x^{2} / 4} d s=\frac{M^{2}}{4 \pi \sqrt{3}}
$$

and hence (A.1) can be written as

or, equivalently,

$$
v(\eta, t)=v_{0}(\eta)+\frac{M^{2}}{4 \pi \sqrt{3}} t^{-1 / 2}(\log t) v_{0}^{\prime}(\eta)+O\left(t^{-1 / 2}\right)
$$

$$
v(\eta, t)=v_{0}\left\{\eta+\frac{M^{2}}{4 \pi \sqrt{3}} t^{-1 / 2} \log t\right\}+O\left(t^{-1 / 2}\right),
$$

which gives the representation (4.18) with (4.20).

The case $p=2$ can be dealt with in a similar way. With this value of $p,(4.13)$ becomes

$$
t \frac{\partial v}{\partial t}-\frac{1}{2}\left(v+\eta \frac{\partial v}{\partial \eta}\right)+t^{-1 / 2}\left\{t \frac{\partial\left(v^{2}\right)}{\partial t}-\frac{p}{2} v^{2}-\frac{\eta}{2} \frac{\partial\left(v^{2}\right)}{\partial \eta}\right\}=\frac{\partial^{2} v}{\partial \eta^{2}}+\frac{\partial\left(v^{2}\right)}{\partial \eta} .
$$

Again we have to expand

$$
v(\eta, t)=v_{0}(\eta)+t^{-1 / 2}(\log t) v_{1}(\eta)+t^{-1 / 2} v_{2}(\eta)+o\left(t^{-1 / 2}\right),
$$

where this time $v_{0}(\eta)$ is given by (4.30). Substituting (A.6) into (A.5), equating terms $O\left(t^{-1 / 2} \log t\right)$ and requiring exponential decay on $v_{1}$ as $\eta \rightarrow \pm \infty$ gives

$$
v_{1}=B v_{0}^{\prime}(\eta) \text {. }
$$

A similar procedure for terms of $O\left(t^{-1 / 2}\right)$ gives the condition on $B$ for exponential decay of $v_{2}$ as $\eta \rightarrow \pm \infty$ as

$$
B=\int_{-\infty}^{\infty} \frac{\left(v_{0}^{2}+s v_{0} v_{0}^{\prime}\right) v_{0}^{\prime}}{v_{0}^{2}} e^{-s^{2} / 4} d s / \int_{-\infty}^{\infty}\left(\frac{v_{0}^{\prime}}{v_{0}}\right)^{2} e^{-s^{2} / 4} d s
$$

Numerical evaluation gives $B=-0-0630$ to four decimal places.

\section{APPENDIX B}

Solution to a boundary-value problem

In this Appendix we construct the solution to the boundary-value problem represented by (4.86) to (4.88) in section 4.2 .2 .

We first show that $U_{0}(\xi)$ is monotonically increasing for $-\infty<\xi<\infty$. To see this we suppose that $\xi_{0}$ is a stationary point of $U_{0}(\xi)$ so that from (4.86) we have, with 
$U_{0}\left(\xi_{0}\right)>0$

$$
U_{0}^{*}=-\frac{p}{(1-p)} U g\left(\xi_{0}\right)<0
$$

so $\xi_{0}$ is a maximum. This implies that $U_{0}(\xi)<U_{0}\left(\xi_{0}\right)$ for all $\xi$ so the boundary condition (4.87) cannot be satisfied, hence $U_{0}$ cannot have a stationary point and thus the monotonicity condition must hold. This result now enables us to change the independent variable from $\xi$ to $U_{0}$. Putting $U_{0}=u$ we write

$$
u^{\prime}(\xi)=y(u)
$$

regarding $u$ as the independent variable with $0<u<\infty$. This reduces (4.86) to the first-order equation

$$
\frac{d y}{d u}=1+\zeta_{0} p u^{p-1}-\frac{p}{(1-p)} \frac{u^{p}}{y} .
$$

Since we are assuming that $u=U_{0}(\xi)$ has a continuous derivative for all $\xi$ then we must have $u^{\prime}(\xi) \rightarrow 0$ as $\xi \rightarrow \infty$ and we must solve (B.2) subject to the initial condition $y(0)=0$. Now near $u=0$

$$
y(u) \sim \zeta_{0} u^{p}+\cdots
$$

and hence from (B.1) we see that the support of $u(\xi)$ must be finite since with $0<p<1$

$$
\int_{0}^{*} \frac{d s}{y(s)}
$$

is bounded. Thus $u(\xi) \geqslant 0$ for $\xi \geqslant \xi_{1}$ and $u(\xi)=0$ for $-\infty<\xi \leqslant \xi_{1}$ where we may take $\xi_{1}=0$ since (4.86) is translationally invariant. To see what conditions hold at $\xi=0$ we integrate (4.86) between zero and $\xi>0$ with $u(0)=u^{\prime}(0)=0$ to give

$$
u^{\prime}(\xi)=\zeta_{0} u^{p}(\xi)+u(\xi)-\frac{p}{(1-p)} \int_{0}^{\xi} u^{p}(s) d s,
$$

whence dividing by $u^{p}(\xi)>0$ we have

$$
\frac{u^{\prime}(\xi)}{u^{\prime}(\xi)}=\zeta_{0}+u^{1-p}(\xi)-\frac{p}{(1-p) u^{p}(\xi)} \int_{0}^{\zeta} u^{p}(s) d s .
$$

Since $u^{p}(\xi)$ is monotonically increasing it follows that

so

$$
\int_{0}^{\xi} u^{p}(s) d s<\xi u^{p}(\xi)
$$

or

at $\xi=0$.

$$
\lim _{\xi \rightarrow 0}\left\{\frac{u^{\prime}(\xi)}{u^{\prime}(\xi)}\right\}=\zeta_{0}
$$

in (B.2) to give

$$
\left\{u^{1-p}(\xi)\right\}^{\prime}=\zeta_{0}(1-p)
$$

We are now in a position to construct the solution. First we put

$$
v=u^{1-p} \text { and } v^{\prime}(\xi)=Y(v)
$$

$$
\frac{d Y}{d v}=\frac{Y-p}{Y}+\frac{p}{(1-p) v}\left\{\zeta_{0}(1-p)-Y\right\},
$$


where from (B.3)

and from (4.87)

$$
\begin{aligned}
Y(0) & =(1-p) \zeta_{0} \\
Y(\infty) & =p .
\end{aligned}
$$

To determine $\zeta_{0}$ and $Y(v)$ we proced as follows. First if we suppose that $\zeta_{0}>p /(1-p)$ then $Y(0)>p$ and, from (B.4), $Y^{\prime}(0)>0$. Now in order for the condition (B.6) to be satisfied there must be a value of $v>0$ for which $Y=\zeta_{0}(1-p)$ and $Y^{\prime}<0$. Clearly from (B.4) such a condition cannot hold for $\zeta_{0}>p /(1-p)$. A similar argument holds if $\zeta_{0}<p /(1-p)$ and so we conclude that

whence the problem becomes

$$
\zeta_{0}=p /(1-p)
$$

$$
\frac{d Y}{d v}=\frac{(Y-p)\{(1-p) v-p Y\}}{v Y(1-p)}, \quad Y(0)=p .
$$

Now there are no solutions which increase from $Y(0)=p$ since $d Y / d v<0$ for $Y<p$ and $Y>(1-p) v / p$. Similarly there are no solutions which decrease from $Y(0)=p$. Thus $Y=p$ is the only solution satisfying $Y(0)=p$ and hence with (B.7) constitutes the unique solution. In the original variables this becomes

$$
U_{0}= \begin{cases}\left\{p\left(\xi-\xi_{1}\right)\right\}^{1 /(1-p)}, & \xi \geqslant \xi_{1}, \\ 0, & \xi<\xi_{1},\end{cases}
$$

which together with (B.7) is the unique solution, to within the arbitrary translation $\xi_{1}$, of the boundary value problem (4.86) to $(4.88)$.

\section{APPENDIX C}

The solution of the initial-value problem for $D=0$

In this Appendix we write down the solution of the equation (2.11) with $D=0$ and initial data (4.17) for $p=\frac{1}{2}$ and $p=\frac{3}{2}$.

1. $p=\frac{1}{2}$. In this case the point $x=-1$, where $u=0$, does not move so there is an expansion wave which connects $u=0$ to $u=1$. In addition a shock initiated at $x=1$ moves to the right with a speed determined by the mass invariance condition. So we have

$$
u(x, t)= \begin{cases}0, & x<-1 \\ \frac{(1+x)^{2}}{4(t-x-1)^{2}}, & -1 \leqslant x \leqslant(2 t / 3)-1 \\ 1, & (2 t / 3)-1 \leqslant x \leqslant(t / 2)+1 \\ 0, & x>(t / 2)+1\end{cases}
$$

This persists until the head of the expansion wave coalesces with the shock at $t=12$ and $x=7$. For $t>12$ we then have

where

$$
u(x, t)= \begin{cases}0, & x<-1, \\ \frac{(1+x)^{2}}{4(t-x-1)^{2}}, & -1 \leqslant x \leqslant S(t), \\ 0, & x>S(t),\end{cases}
$$

$$
S(t)=4(t+4)^{1 / 2}-9, \quad t \geqslant 12 .
$$


In terms of the outer variable of section 4.1 .3 , namely $\eta=x / t^{1 / 2}$, we have

with

$$
v(\eta, t)= \begin{cases}0, & \eta<-t^{-1 / 2}, \\ \frac{\left(\eta+t^{-1 / 2}\right)^{2}}{4\left(1-\eta t^{-1 / 2}-t^{-1}\right)^{2}}, & -t^{-1 / 2} \leqslant x \leqslant \eta_{2}(t), \\ 0, & x \geqslant \eta_{2}(t),\end{cases}
$$

$$
\eta_{2}(t)=4\left(1+4 t^{-1 / 2}\right)^{1 / 2}-9 t^{-1 / 2} .
$$

2. $p=\frac{3}{2}$. Here the point $x=1$ translates to the right with unit speed so we put

$$
X=x-t
$$

and the leading edge of the wave, where $u=0$, is located at $X=1$. There is an expansion wave which links $u=0$ and $u=1$, together with a shock which moves to the left in the $(X, t)$-plane from $X=-1$ according to mass conservation in the wave.

Hence the solution can be written

$$
u(X, t)= \begin{cases}0, & X<-1-(t / 5), \\ 1, & -1-(t / 5) \leqslant X<1-(3 t) / 5, \\ \frac{4(1-X)^{2}}{9(t-1+X)^{2}}, & 1-(3 t) / 5<X \leqslant 1, \\ 0, & X \geqslant 1 .\end{cases}
$$

The solution persists until $t=20$, at which time the shock and expansion waves coalesce. For $t \geqslant 20$ we then have

$$
u(X, t)= \begin{cases}0, & X<X_{1}(t), \\ \frac{4(1-X)^{2}}{9(X+t-1)^{2}}, & X_{1}(t) \leqslant X \leqslant 1, \\ 0, & X>1,\end{cases}
$$

where $X_{1}(t)$ is given by the solution of a cubic. In terms of the outer variable of section 4.1.2, namely $\eta=X / t^{2 / 3}$, we have

where

$$
v(\eta, t)= \begin{cases}0, & \eta<\eta_{1}(t), \\ \frac{4\left(t^{-2 / 3}-\eta\right)^{2}}{9\left(1+\eta t^{-1 / 3}-t^{-1}\right)^{2}}, & \eta_{1}(t) \leqslant \eta \leqslant t^{-2 / 3}, \\ 0, & \eta>t^{-2 / 3},\end{cases}
$$

$$
\begin{gathered}
\eta_{1}(t)=t^{-2 / 3}(12(u+v)-8), \quad u=\left(-q+\left(q^{2}+p^{3}\right)^{t}\right)^{1 / 3}, \quad v=-p / u, \\
p=t / 8-9 / 16 \text { and } \quad q=t^{2} / 128-(9 t) / 64+27 / 64 .
\end{gathered}
$$

